\title{
Digital Architectures for UWB Beamforming Using 2D IIR Spatio-Temporal Frequency-Planar Filters
}

\author{
Soumya Kondapalli, ${ }^{1}$ Arjuna Madanayake, ${ }^{1,2}$ and Len Bruton ${ }^{2}$ \\ ${ }^{1}$ Electrical and Computer Engineering, The University of Akron, Akron, OH 44325-3904, USA \\ ${ }^{2}$ Electrical and Computer Engineering, The University of Calgary, Calgary, AB, Canada T2N 1N4
}

Correspondence should be addressed to Arjuna Madanayake, arjuna@uakron.edu

Received 14 May 2012; Accepted 19 June 2012

Academic Editor: Marco Antonio Panduro-Mendoza

Copyright (C) 2012 Soumya Kondapalli et al. This is an open access article distributed under the Creative Commons Attribution License, which permits unrestricted use, distribution, and reproduction in any medium, provided the original work is properly cited.

\begin{abstract}
A design method and an FPGA-based prototype implementation of massively parallel systolic-array VLSI architectures for 2ndorder and 3rd-order frequency-planar beam plane-wave filters are proposed. Frequency-planar beamforming enables highlydirectional UWB RF beams at low computational complexity compared to digital phased-array feed techniques. The array factors of the proposed realizations are simulated and both high-directional selectivity and UWB performance are demonstrated. The proposed architectures operate using 2's complement finite precision digital arithmetic. The real-time throughput is maximized using look-ahead optimization applied locally to each processor in the proposed massively-parallel realization of the filter. From sensitivity theory, it is shown that 15 and 19-bit precision for filter coefficients results in better than 3\% error for 2nd- and 3rdorder beam filters. Folding together with Ktimes multiplexing is applied to the proposed beam architectures such that throughput can be traded for $K$-fold lower complexity for realizing the 2-D fan filter banks. Prototype FPGA circuit implementations of these filters are proposed using a Virtex 6 xc6vsx475t-2ff1759 device. The FPGA-prototyped architectures are evaluated using area $(A)$, critical path delay $(T)$, and metrics $A T$ and $A T^{2}$. The $L 2$ error energy is used as a metric for evaluating fixed-point noise levels and the accuracy of the finite precision digital arithmetic circuits.
\end{abstract}

\section{Introduction}

Radio-frequency (RF) two-dimensional (2D) infinite impulse response (IIR) space-time (ST) plane-wave frequencyplanar beam filters [1] have potential applications in ultrawideband (UWB) directional filtering of propagating electromagnetic far-field plane-waves. Such plane-wave filters achieve highly directional beamforming for aperture array applications. The proposed beam filters are designed using the concept of frequency-planar resonant $2 \mathrm{D}$ inductorcapacitor (LC) ladder network prototypes having resistive terminations [2]. For example, UWB beam filters can be employed in radar [3], wireless communications [4], radio astronomy [5], and electromagnetic imaging, and sensing [6]. Furthermore, new applications have been proposed in cognitive radio towards enhanced access to radio spectrum (EARS) [7] which requires sensitive spectrum sensing in both space and time domains [8], in turn leading to a strong need for low-complexity directional filters capable of real-time RF operation [9].

High attenuation in the stop-band region as well as a sharp transition from filter passband to stop-band is greatly desired in high-performance beamforming systems because such characteristics are important for achieving a better approximation to the ideally "brick-wall" type transition between main beam and stop-band null of the array pattern. The sharpness of the transition from passband (main beam) to stop-band (null region) of the aperture array factor directly depends on the order of the transfer function of the spatio-temporal filter that is employed for UWB beamforming. The primary objective of this work is to explore the realtime hardware architectures that are necessary for realizing frequency-planar beam plane-wave filters corresponding to 2nd- and 3rd-order 2D passive LC ladder low-pass networks. The proposed architectures are an extension of the elementary 1st-order hardware architecture described in [10]. Here, 
we propose novel massively parallel digital architectures with detailed design equations and complexity studies for beamforming networks based on LC-ladder prototypes of order 2 and 3 having significantly better UWB directionality compared to available 1st-order realizations [10].

The 2D frequency-planar beam filters are both practical bounded-input and bounded-output (practical-BIBO) stable [11] and structurally stable under zero initial conditions (ZICs) and can be designed for low computational complexity. We propose massively parallel systolic-array VLSI architectures containing identical and locally interconnected parallel processing core modules (PPCMs) for 2 nd-order and 3rd-order beam filters. The proposed architectures are finegrain pipelined using both inter-PPCM and intra-PPCM registers in order to reduce the critical path delays (CPDs) for achieving maximum clock frequency and temporal bandwidth. The main reason to pipeline the filters is to achieve higher throughputs required for real-time filtering of $2 \mathrm{D}$ RF signals derived from time synchronously sampled UWB uniform linear arrays (ULAs) of antenna elements. The proposed systolic-array architectures achieve real-time RF plane-wave filtering at a throughput of one-frame-per-clockcycle (OFPCC). The frame sampling rate of the beamformer is equal to the clock frequency $F_{\text {clock }} \leq 1 / \Delta T_{\text {cpd }} \mathrm{Hz}$.

An approximately frequency-independent beam shape over an UWB frequency range is obtained by employing a fan filter bank configuration where each subband of the fan filter bank consists of a temporally bandpass frequencyplanar beam filter [12]. For the best real-time throughput, each subband of the fan filter bank [12] may be realized using dedicated massively parallel systolic-array processors. However, this technique results in high circuit complexity due to the high degree of parallelism. In this work, we trade throughput for lower circuit complexity by employing a folded architecture for the realization of the fan filter bank. The hardware design approach of folding leads to the time interleaving of multiple 2D filters. Time multiplexing of filter coefficients in folded hardware allows different filters [13] to share arithmetic hardware thereby reducing the number of multipliers and adders. $K$-times folding results in $K$-fold utilization of hardware at the cost of a $K$-fold loss in ULA linear frame-rate [14].

\section{Review: Beam Filter Design}

2.1. Review of UWB Beamforming. Beamforming enables array factors (AFs) [15] with steerable directional sensitivities which are used to enhance/reject plane-wave signals [1622]. Real-time UWB beamforming benefits both engineering and scientific applications such as radar [23-26], navigation [27], wireless communications [18, 28-31], cognitive radio $[32,33]$, frequency-agile antennas [34], radio astronomy and RF space imaging [35-45], microwave imaging and remote sensing applications [46-48]. Radio telescopes [49-52] such as the square kilometer array (SKA) [53-55] requires algorithms for real-time UWB beamforming $(70 \mathrm{MHz}-1.4 \mathrm{GHz})$ with multiple beams [56].

Aperture arrays [16-22, 42, 57-65] enable directional enhancement of plane-wave signals [15] (such as signals from deep space, wireless base stations, etc.). The electronically steerable nature of the beam direction and size makes beamforming an essential signal processing task in many applications including radio astronomy [35-45, 49$52,66-88]$, radar and navigation [23-27], and broadband wireless communication systems [18, 29-31]. Algorithms for beamforming require low-noise amplification [69-71] of the analog signals from the aperture arrays.

In digital beamforming, an array of time synchronous A/D converters [72] leads to the discrete-time signals. Such algorithms are based on either time domain delay-andsum (DAS) or frequency domain phased array feed (PAF) techniques $[16,19,73]$. In delay-and-sum-based systems, element signals are delayed and added coherently to form a beam. True time delays for each antenna are found for a particular beam direction and inter antenna spacing and are realized as tapped delay lines $[74,75]$. In finite impulse response (FIR) beamforming [60, 61, 76, 77], the time delays are implemented as FIR digital filters. This method has high computational intensity compared to the proposed IIR technique. In frequency domain PAF beamforming [57, 58, 65], the digitized DAA signals are converted to frequency domain by evaluating the temporal fast Fourier transform (FFT) $[78,79]$. The FFT bins are subsequently multiplied by a complex weight and coherently summed to achieve frequency domain beamforming.

2.2. Principle of $2 D$ IIR Beamforming. Figure 1 shows the overview of the proposed UWB digital beamforming system. The digital input signals of the proposed massively parallel systolic-array architectures are obtained by amplifying and low-pass filtering the continuous-time (i.e., analog) signals from UWB low-noise antennas. Typical choices for the RF sensing antenna are Vivaldi or BAVA antennas; however, other types of broadband antennas such as biconical antennas may also be employed.

UWB beamforming is implemented here using an array of antennas placed at uniform distance along $x$-axis. The principle of UWB beam filtering $[2,80]$ is to enhance the spectrum $W_{\text {pass }}\left(j \omega_{1}, j \omega_{2}, \psi\right)$ of spatio-temporal plane wave $w_{\text {pass }}\left(\Delta x n_{1}, c \Delta T_{s} n_{2}, \psi\right)$ propagating with a desired direction of arrival (DOA) of $\psi$ while simultaneously attenuating all undesired signals $w_{\text {stop }}\left(\Delta x n_{1}, c \Delta T_{s} n_{2}, \psi\right)$ with spectrum $W_{\text {stop }}\left(j \omega_{1}, j \omega_{2}, \psi\right)$ which lie outside the passband of the filters.

From Nyquist sampling theorem, $\Delta x=c \Delta T_{s}$, where $\Delta T_{s}$ is the temporal sampling period and $c \approx 3 \cdot 10^{8} \mathrm{~ms}^{-1}$ is the speed of light in air [81]. The plane-wave signal of interest is denoted by $w_{\text {pass }}(\cdot)$ while interference signals are denoted by $w_{\text {stop }}(\cdot)$, respectively. The $2 \mathrm{D}$ sampled input signal is of the form

$$
w\left(n_{1}, n_{2}\right)=w_{\text {pass }}\left(l_{k}\right)+\sum_{k=1}^{M} w_{\text {stop }}\left(l_{k}\right)+n_{v}\left(n_{1}, n_{2}\right),
$$

where $l_{k}=-n_{1} \Delta x \sin \theta_{k}+n_{2} c \Delta T_{s}, 0 \leq n_{2}$, and $0 \leq n_{1}<$ $N_{1}$ corresponding to the number of elements in the ULA. Additive white Gaussian noise (AWGN) in the 2D signal is represented by $n_{v}\left(n_{1}, n_{2}\right)$. The spatial DOA [82] is measured 


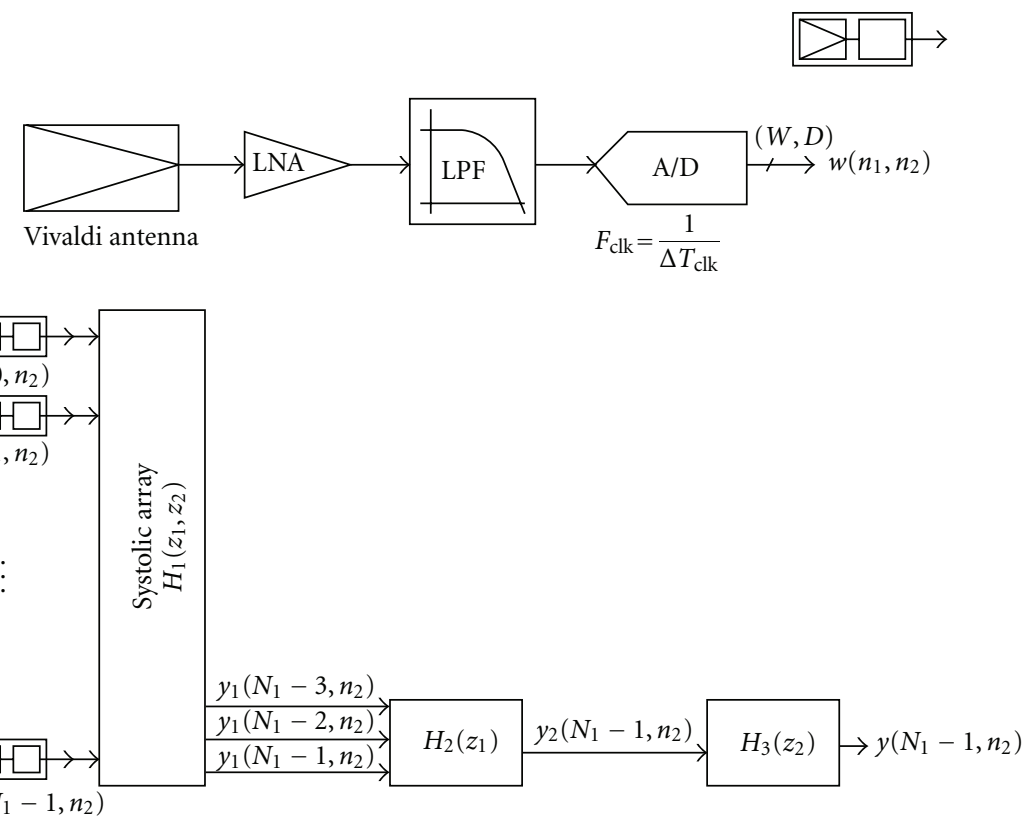

FiguRe 1: RF front-end for a single array element showing antenna, LNA, low-pass filter, and A/D converters. The smart antenna array consists of several such mixed-signal RF circuits for electromagnetic signal sampling at real-time RF rates.

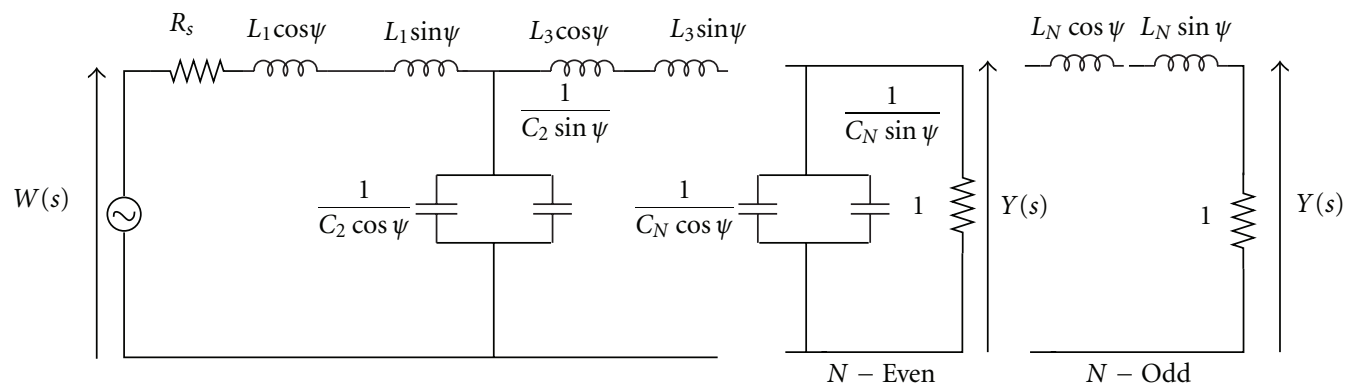

FIGURE 2: 2D passive LRC circuit for $N$ th-order filter.

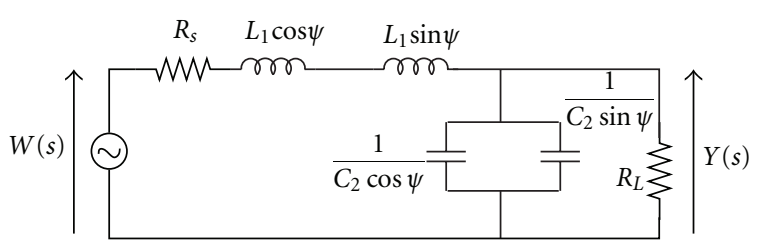

FIgURE 3: 2D passive LRC circuit for 2nd-order filter.

from the broadside direction of the ULA and is denoted by $\theta$ where $0 \leq \theta \leq 90^{\circ}$ and $\psi$ is the corresponding space-time DOA, $0 \leq \psi \leq 45^{\circ}$,

$$
\psi_{k}=\arctan \left(\frac{\Delta x \sin \theta_{k}}{c \Delta T_{s}}\right)
$$

2.3. Review of 2D Plane-Wave Beam Filters. The 2D IIR plane-wave beam filters can be synthesized [83] using 2D LC network prototypes. The Laplace transfer function derived

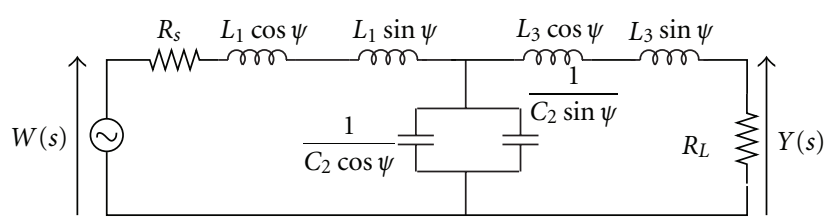

FIGURE 4: 2D passive LRC circuit for 3rd-order filter.

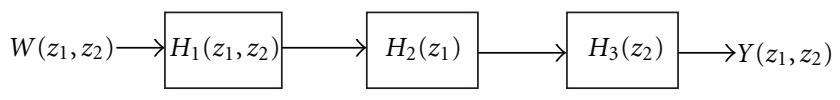

FIGURE 5: Cascaded form of 2D Nth-order beam filter.

from the network is found and subsequently converted to the $2 \mathrm{D} \mathbf{z}$-domain using the complex map of bilinear transformation. The resulting $\mathbf{z}$-domain transfer function leads to a computable 2D difference equation of the filter enabling 


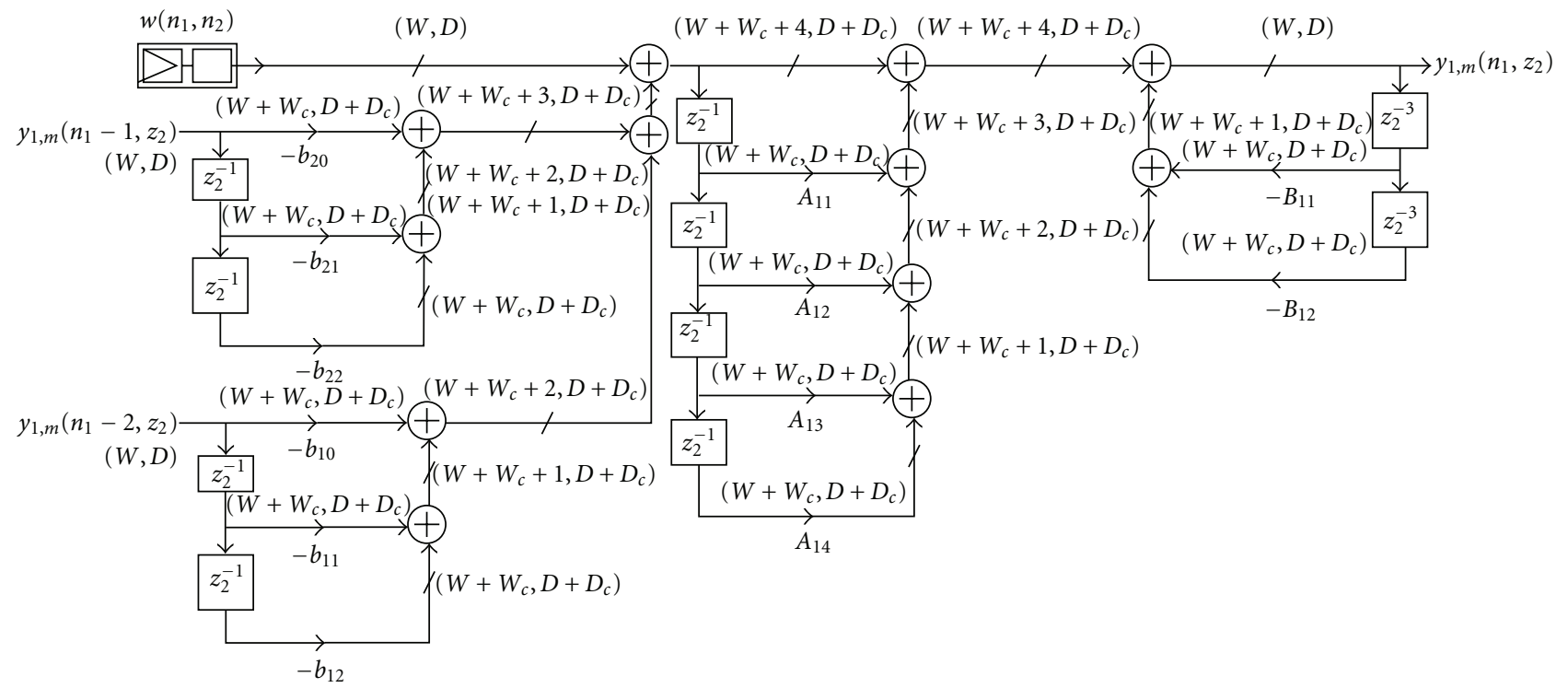

Figure 6: Architecture of $H_{1, m}^{\prime}\left(n_{1}, z_{2}\right)$ of 2D 2nd-order beam filter with 3-stage scattered look-ahead pipelining.

real-time digital VLSI realization using RF rate systolicarray processors. Examples of a 1st-order beam filter were previously investigated in [10]. An example of 1st-order 3D IIR cone filter-bank was first proposed in [12].

We here propose a 2 nd-order plane-wave filter hardware having useful applications as a building block for achieving fan filter banks. A sharper transition required for aperture arrays can be obtained with higher order filters. Further, we propose 3rd-order plane-wave filter hardware and estimate both complexity, quantization noise level, and performance. The sharper transition (roll-off) for 3rd-order filter in comparison to the 2nd-order filter is demonstrated.

Let the 1D input-output Laplace transfer function of a classical resistively-terminated $N$ th-order LC ladder low-pass network shown in Figure 2 be given by

$$
T(s)=\frac{Y(s)}{W(s)} .
$$

The above equation can be converted to a 2D Laplace equation by applying frequency-planar transformation, $s=$ $s_{1} \cos \psi+s_{2} \sin \psi$ to (3) to obtain $T\left(s_{1}, s_{2}\right)$ :

$$
T\left(s_{1}, s_{2}\right)=\frac{Y\left(s_{1}, s_{2}\right)}{W\left(s_{1}, s_{2}\right)} .
$$

$T\left(s_{1}, s_{2}\right)$ is mapped to the $2 \mathrm{D} \mathbf{z}$-domain by applying bilinear transformation to get

$$
\begin{aligned}
H\left(z_{1}, z_{2}\right) & =\frac{N\left(z_{1}, z_{2}\right)}{D\left(z_{1}, z_{2}\right)} \\
& =\left.T\left(s_{1}, s_{2}\right)\right|_{s_{k}=\left(1-z_{k}^{-1}\right) /\left(1+z_{k}^{-1}\right), k=1,2} .
\end{aligned}
$$

Note that the above design equations are limited to filter passbands that exist in the second and fourth quadrants of the $2 \mathrm{D}$ frequency space. Given that filter stability requires all components to be nonnegative [2], for beamforming in quadrants one and three (i.e., $-\pi / 2 \leq \theta \leq 0$ ), we use nonnegative values in each branch impedance and shunt admittance of the prototype while mirroring the input array signal spatially because $w(-x, c t) \Longleftrightarrow W\left(-j \omega_{1}, j \omega_{2}\right)$ such that the passband spectra now fall within quadrants two and four of the frequency space [2].

To obtain 2D difference equations, we apply the inverse $Z$-transform to the above function under ZICs, leading to $N$ th-order plane-wave beam filter realizations having general form [2]

$$
\begin{aligned}
y\left(n_{1}, n_{2}\right)= & \sum_{i=0}^{N} \sum_{j=0}^{N} a_{i j} w\left(n_{1}-i, n_{2}-j\right) \\
& -\sum_{i=0}^{N} \sum_{j=0}^{N} b_{i j} y\left(n_{1}-i, n_{2}-j\right) .
\end{aligned}
$$

For feed-back paths $i+j \neq 0,0 \leq n_{1}<N_{1}$ and $0 \leq n_{2}$. The frequency response of the Laplace domain prototype is obtained by evaluating $T\left(j \omega_{1}, j \omega_{2}\right)$. The frequency response of the digital realization is obtained in closed form by evaluating $H\left(e^{j \omega_{1}}, e^{j \omega_{2}}\right)$ [2]. That is, on the $2 \mathrm{D}$ unit bicircle $\left|z_{1}\right|=\left|z_{2}\right|=1$. The frequency response may be verified by computing the $2 \mathrm{D}$ discrete Fourier transform (DFT) of the unit impulse response $h\left(n_{1}, n_{2}\right)$ and comparing with the closed-form response $H\left(e^{j \omega_{1}}, e^{j \omega_{2}}\right)$.

\section{Systolic-Array Architecture of 2D IIR Filters}

3.1. Difference Equations. In our example design for 2ndorder plane-wave filter shown in Figure 3, we employ parameters $R_{s}=1, L_{1}=1.4142, C_{2}=1.4142$ [83]. For our example of 3rd-order beam filter shown in Figure 4 , the design parameters are $R_{s}=1, L_{1}=1, C_{2}=2, L_{3}=1$ and the desired DOA is $\psi=10^{\circ}$ [83]. Table 1 gives the feedback 


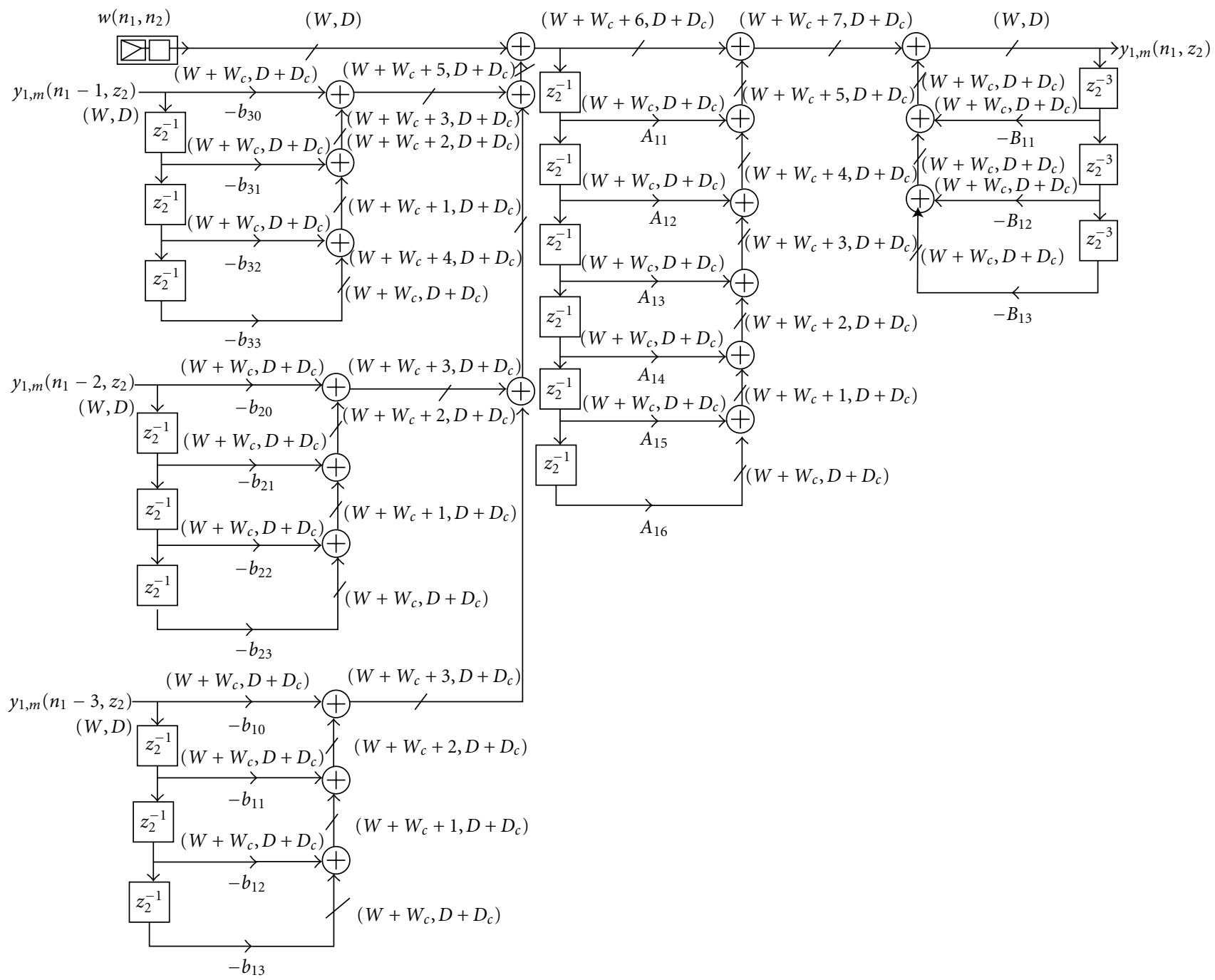

FIGURE 7: Architecture of $H_{1, m}^{\prime}\left(n_{1}, z_{2}\right)$ of 2D 3rd-order beam filter with 3-stage scattered look-ahead pipelining.

coefficients of the filter for a 1st-order, 2nd-order, and 3rdorder plane-wave beam filters [84].

3.2. Partially Separable Signal Flow Graphs. In order to reduce digital implementation complexity, we first separate the $2 \mathrm{D} \mathbf{z}$-domain transfer function of the beam filters into separable and nonseparable subfilters. These subfilters correspond to spatial, temporal, and spatio-temporal prototype networks. Therefore, the transfer functions are cascaded to form the final 2D filter as shown in Figure 5. Following [10] to higher-order filters, we propose that the nonseparable function $H_{1}\left(z_{1}, z_{2}\right)$ be realized employing a systolic-array consisting of $N_{1}$ similar parallel processing core modules (PPCMs) which are interconnected to each other such that spatio-temporal feed-forward and feedback paths required for recursive computation of the filter are realized using $2 \mathrm{D}$ difference equations.
The input RF waves received by the each antenna in the ULA is passed through LNAs, low pass filtered, samples, and quantized within each ADC. The sampled digital signals are connected to the PPCM input ports. The corresponding outputs are sent through $H_{2}\left(z_{1}\right)$ and $H_{3}\left(z_{2}\right)$ as shown in Figure 1. The function $H_{2}\left(z_{1}\right)$ is implemented as filter with spatial delays and $H_{3}\left(z_{2}\right)$ is implemented using temporal delays:

$$
\begin{gathered}
H\left(z_{1}, z_{2}\right)=H_{1}\left(z_{1}, z_{2}\right) H_{2}\left(z_{1}\right) H_{3}\left(z_{2}\right), \\
H_{1}\left(z_{1}, z_{2}\right)=\frac{1}{1+\sum_{i=0}^{N} \sum_{j=0}^{N} b_{i j} z_{1}^{-i} z_{2}^{-j}} \\
H_{2}\left(z_{1}\right)=\left(1+z_{1}^{-1}\right)^{N} \\
H_{3}\left(z_{2}\right)=\left(1+z_{2}^{-1}\right)^{N} .
\end{gathered}
$$




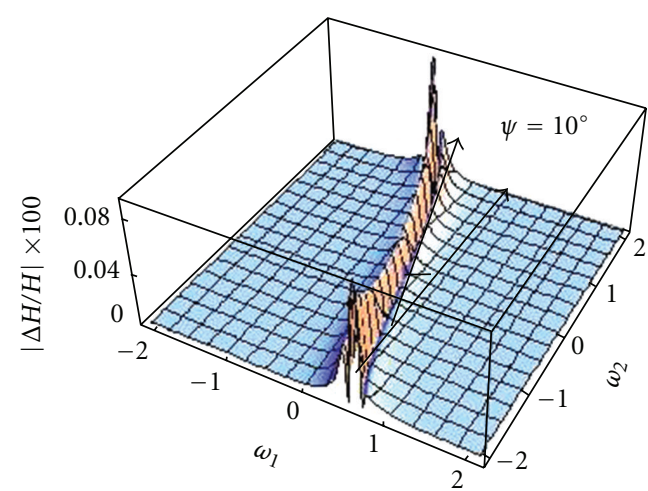

(a) Relative error in $H$ of 2 nd-order filter for $W_{c}=20$

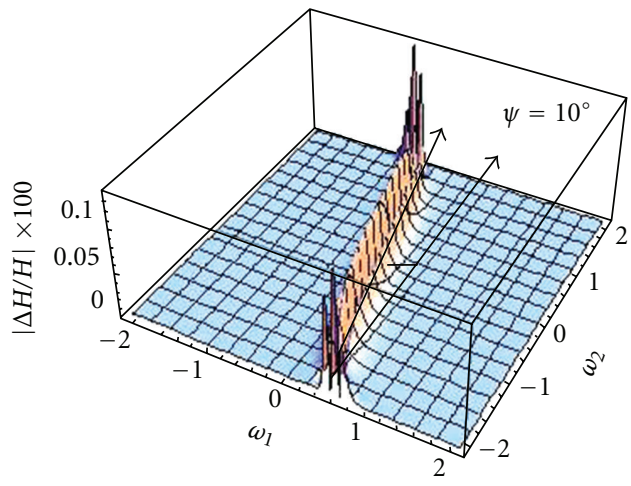

(c) Relative error in $H$ of 3 rd-order filter for $W_{c}=23$

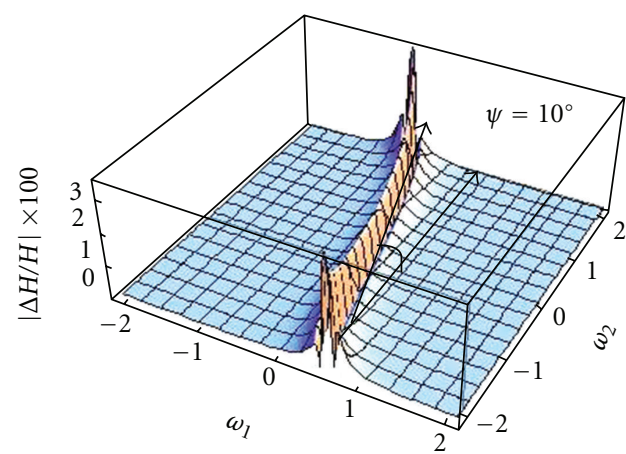

(b) Relative error in $H$ of 2nd-order filter for $W_{c}=15$

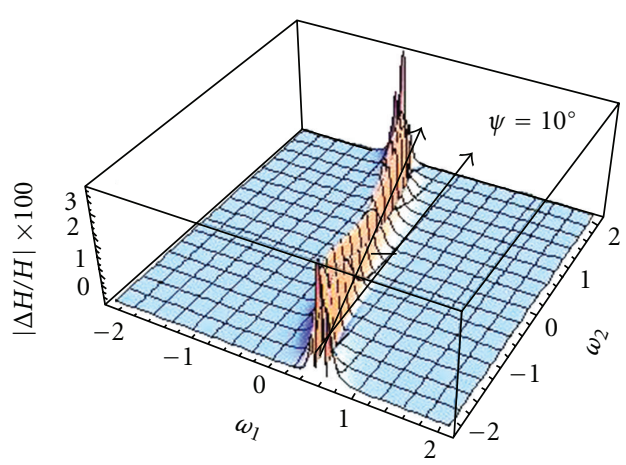

(d) Relative error in $H$ of 3rd-order filter for $W_{c}=19$

FIGURE 8: Relative error in $H\left(z_{1}, z_{2}\right)$ with respect to the respective coefficients of 2nd-order and 3rd-order plane-wave filters.

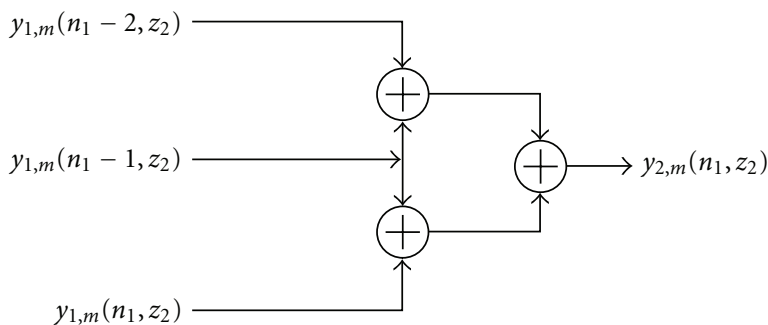

FIGURE 9: Architecture of $H_{2}\left(z_{1}\right)$ of 2D 2nd-order beam filter.

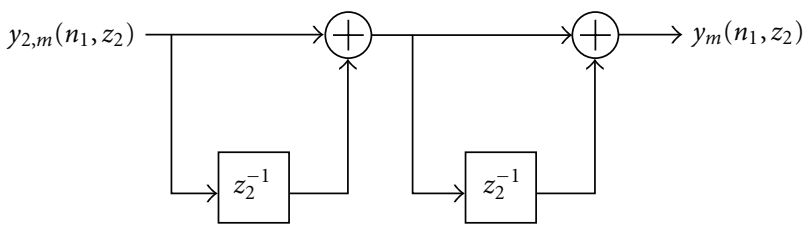

FIgURE 10: Architecture of $\mathrm{H}_{3}\left(z_{2}\right)$ of 2D 2nd-order beam filter.

Zero-initial conditions (ZICs) of the filter along both discrete space and time dimensions are defined by

$$
\begin{aligned}
& w\left(-\Delta x, c \Delta T_{s} n_{2}\right)=w\left(\Delta x n_{1},-c \Delta T_{s}\right) \equiv 0, \\
& y\left(-\Delta x, c \Delta T_{s} n_{2}\right)=y\left(\Delta x n_{1},-c \Delta T_{s}\right) \equiv 0 .
\end{aligned}
$$

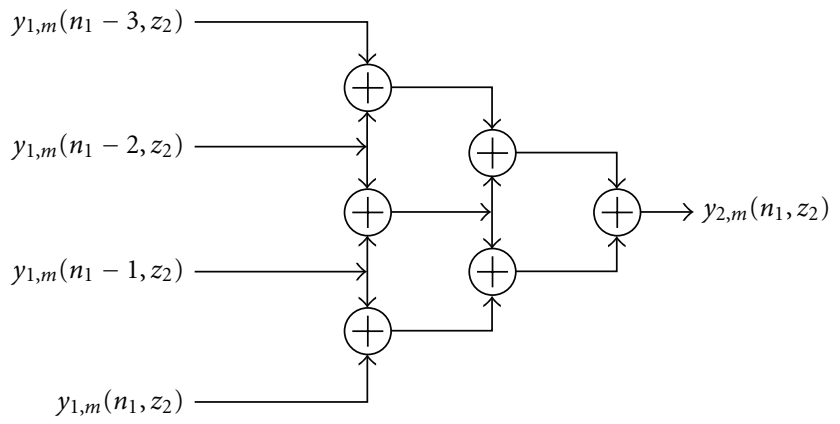

Figure 11: Architecture of $H_{2}\left(z_{1}\right)$ of 2D 3rd-order beam filter.

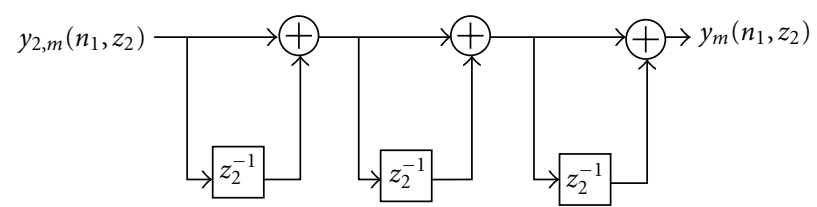

Figure 12: Architecture of $H_{3}\left(z_{2}\right)$ of 2D 3rd-order beam filter.

The temporal ZICs $[10,11]$ are provided to the design by preloading the input values with zeros. Spatial ZICs are provided by connecting constant value zero as previous state inputs to the first PPCM. The function $H_{1}\left(z_{1}, z_{2}\right)$ for an $N$ thorder plane-wave beam filter given in (7) is implemented as 
TABLE 1: Filter design equations showing algebraically defined filter coefficients as functions of LC ladder prototype network parameters and steered angle $\tan \psi=\sin \theta$. Here, $\cdot$ refers to dot product between vectors.

\begin{tabular}{|c|c|c|}
\hline Order of filter & $\begin{array}{l}\text { Arbitrary coefficients } \\
e_{m}\end{array}$ & $\begin{array}{l}\text { Coefficients } \\
b_{i j}\end{array}$ \\
\hline 1 & $\begin{array}{l}L_{i}=L \cos \psi \\
L_{j}=L \sin \psi \\
e_{1}=1+R_{s} \\
e_{2}=L_{i}, e_{3}=L_{j}\end{array}$ & $\begin{array}{l}b_{i j}=\hat{e} \cdot \hat{\beta}_{i j}, b_{00}=0 \\
\hat{e}=\left(e_{1}, e_{2}, e_{3}\right) / \epsilon \\
\epsilon=e_{1}+e_{2}+e_{3} \\
\beta_{01}=(1,1,-1) \\
\beta_{10}=(1,-1,1) \\
\beta_{11}=(1,-1,-1)\end{array}$ \\
\hline 2 & $\begin{array}{l}L_{i}=L_{1} \cos \psi, \\
L_{j}=L_{1} \sin \psi, \\
C_{i}=C_{2} \cos \psi, \\
C_{j}=C_{2} \sin \psi \\
e_{1}=1+R_{s}, \\
e_{2}=L_{i}+R_{s} C_{i}, \\
e_{3}=L_{j}+R_{s} C_{j}, \\
e_{4}=L_{i} C_{i}, e_{5}=L_{j} C_{j}, \\
e_{6}=L_{i} C_{j}+L_{j} C_{i} .\end{array}$ & $\begin{array}{l}b_{i j}=\hat{e} \cdot \hat{\beta}_{i j}, b_{00}=0 \\
\hat{e}=\left(e_{1}, e_{2}, e_{3}, e_{4}, e_{5}, e_{6}\right) / \epsilon \\
\epsilon=e_{1}+e_{2}+e_{3}+e_{4}+e_{4}+e_{6} \\
\beta_{01}=(2,2,0,2,-2,0) \\
\beta_{02}=(1,1,-1,1,1,-1) \\
\beta_{10}=(2,0,2,-2,2,0) \\
\beta_{11}=(4,0,0,-4,-4,0) \\
\beta_{12}=(2,0,-2,-2,2,0) \\
\beta_{20}=(1,-1,1,1,1,-1) \\
\beta_{21}=(2,-2,0,2,-2,0) \\
\beta_{22}=(1,-1,-1,1,1,1)\end{array}$ \\
\hline 3 & $\begin{array}{l}L_{i}=L_{1} \cos \psi, \\
L_{j}=L_{1} \sin \psi, \\
C_{i}=C_{2} \cos \psi, \\
C_{j}=C_{2} \sin \psi, \\
L_{k}=L_{3} \cos \psi, \\
L_{l}=L_{3} \sin \psi, \\
e_{1}=1+R_{s}, \\
e_{2}=L_{i}+L_{k}+R_{s} C_{i}, \\
e_{3}=L_{j}+L_{l}+R_{s} C_{j}, \\
e_{4}=L_{i} C_{i}+L_{k} C_{i}, \\
e_{5}=L_{j} C_{j}+L_{l} C_{j}, \\
e_{6}=L_{i} C_{j}+L_{j} C_{i}+L_{k} C_{j}+L_{l} C_{i}, \\
e_{7}=L_{i} L_{k} C_{i}, e_{8}=L_{j} L_{l} C_{j}, \\
e_{9}=L_{i} L_{k} C_{j}+L_{i} L_{l} C_{i}+L_{j} L_{k} C_{i}, \\
e_{10}=L_{i} C_{i} L_{l}+L_{j} L_{k} C_{j}+C_{i} L_{j} L_{l} .\end{array}$ & $\begin{array}{l}b_{i j}=\hat{e} \cdot \hat{\beta}_{i j}, b_{00}=0, \\
\hat{e}=\left(e_{1}, e_{2}, e_{3}, e_{4}, e_{5}, e_{6}, e_{7}, e_{8}, e_{9}, e_{10}\right) / \epsilon \\
\epsilon=e_{1}+e_{2}+e_{3}+e_{4}+e_{5}+e_{6}+e_{7}+e_{8}+e_{9}+e_{10} \\
\beta_{01}=(3,3,1,3,-1,1,3,-3,1,-1) \\
\beta_{02}=(3,3,-1,3,-1,-1,3,3,-1,-1) \\
\beta_{03}=(1,1,-1,1,1,-1,1,-1,-1,1) \\
\beta_{10}=(3,1,3,-1,3,1,-3,3,-1,1) \\
\beta_{11}=(9,3,3,-3,-3,1,-9,-9,-1,-1) \\
\beta_{12}=(9,3,-3,-3,-3,-1,-9,9,1,-1) \\
\beta_{13}=(3,1,-3,-1,3,-1,-3,-3,1,1) \\
\beta_{20}=(3,-1,3,-1,3,-1,3,3,-1,-1) \\
\beta_{21}=(9,-3,3,-3,-3,-1,9,-9,-1,1) \\
\beta_{22}=(9,-3,-3,-3,-3,1,9,9,1,1) \\
\beta_{23}=(3,-1,-3,-1,3,1,3,-3,1,-1) \\
\beta_{30}=(1,-1,1,1,1,-1,-1,1,1,-1) \\
\beta_{31}=(3,-3,1,3,-1,-1,-3,-3,1,1) \\
\beta_{32}=(3,-3,-1,3,-1,1,-3,3,-1,1) \\
\beta_{33}=(1,-1,-1,1,1,1,-1,-1,-1,-1)\end{array}$ \\
\hline
\end{tabular}

$N_{1}$ similar interconnected PPCMs. Realization of $H_{1}\left(z_{1}, z_{2}\right)$ for a 2nd-order filter is shown in Figure 6 and for 3rd-order filter as shown in Figure 7.

3.3. Fixed-Point Arithmetic and Quantization Effects. Signed fractional numbers are quantized in a finite precision digital representation, which in this case is based on the two's complement format. Hence, we quantize the coefficients of $2 \mathrm{D}$ digital IIR filters using signed fixed-point arithmetic. The size of registers is designed such that $(W, D)$ being the size of the input signal where $W$ is the total size of the two's complement number and $D$ is the binary point location counted from the rightmost position. Also, we use $\left(W_{c}, D_{c}\right)$ as the size of the coefficients of the filter. The fixed-point finite register size of multipliers has been designed at $(W+$ $\left.W_{c}, D+D_{c}\right)$. The size of each digital signal representation after each multiplier and adder has been marked at every point in the realization of $H_{1, m}^{\prime}\left(n_{1}, z_{2}\right)$. The output signal of a given PPCM is provided as one of the inputs to the next PPCM; this requires that the size of the output signal of each PPCM be the same as the size of the input port in the neighbouring PPCM.

3.4. Beam Sensitivity to Filter Coefficient Precision. Firstorder sensitivity gives a measure of error associated with perturbations in coefficients of the filter. The sensitivity of 


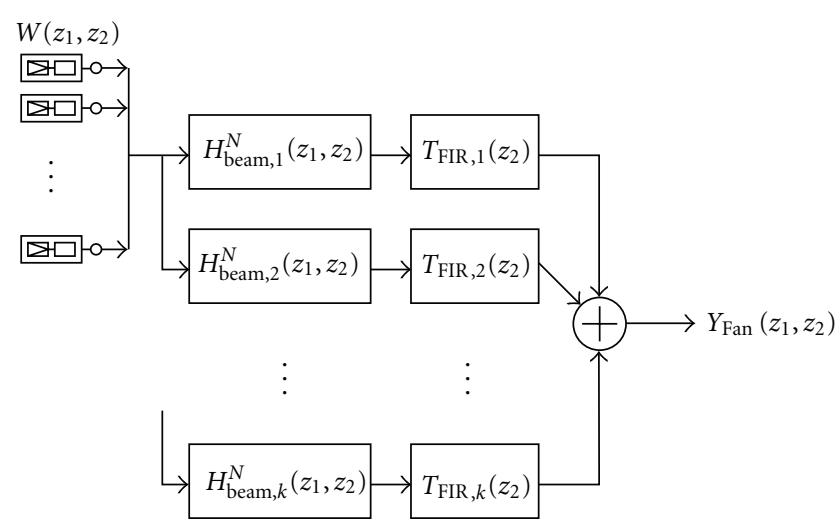

Figure 13: Overview of a K-time multiplexed filter bank architecture [12].

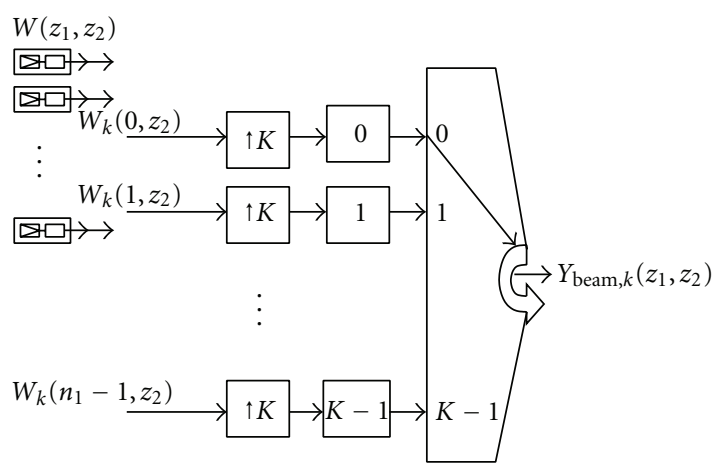

FIgURE 14: Overview of time multiplexing $K$-beam filters.

the $2 \mathrm{D}$ beam transfer function of the filter due to changes in the values of the coefficients that results due to quantization is studied next. We consider 1st-order sensitivity function to find the magnitude error of the design [85]. The sensitivity function for $2 \mathrm{D}$ beam function $H\left(z_{1}, z_{2}\right)$ is given by

$$
S_{b_{i j}}^{H\left(e^{\left.j \omega_{1}, e^{j \omega_{2}}\right)}\right.}=\frac{-b_{i j}}{D\left(z_{1}, z_{2}\right)} \cdot z_{1}^{-i} z_{2}^{-j} .
$$

Sensitivity helps to find relative error in transfer function with respect to a coefficient. If $b_{i j}$ is a coefficient of the filter, then the relative error in the transfer function of the filter due to perturbations in $b_{i j}$ is given by

$$
\frac{\Delta H}{H}=S_{b_{i j}}^{H}\left(\frac{\Delta b_{i j}}{b_{i j}}\right) .
$$

The gain sensitivity in $\left|H\left(e^{j \omega_{1}}, e^{j \omega_{2}}\right)\right|$ is computed using

$$
S_{b_{i j}}^{G\left(\omega_{1}, \omega_{2}\right)}=\operatorname{Real}\left(S_{b_{i j}}^{\mid H\left(e^{\left.j \omega_{1}, e^{j \omega_{2}}\right) \mid}\right)}\right) .
$$

The gain sensitivity in $\left|H\left(e^{j \omega_{1}}, e^{j \omega_{2}}\right)\right|$ due to fixed-point errors in all the coefficients of the filter is given by the summation of the gain sensitivities with respect to each coefficient [85]. We consider nonuniform error in the coefficients given by $\epsilon_{i}$. The relative error in $H\left(z_{1}, z_{2}\right)$ with respect to different sizes of coefficients for 2 nd-order and 3rd-order

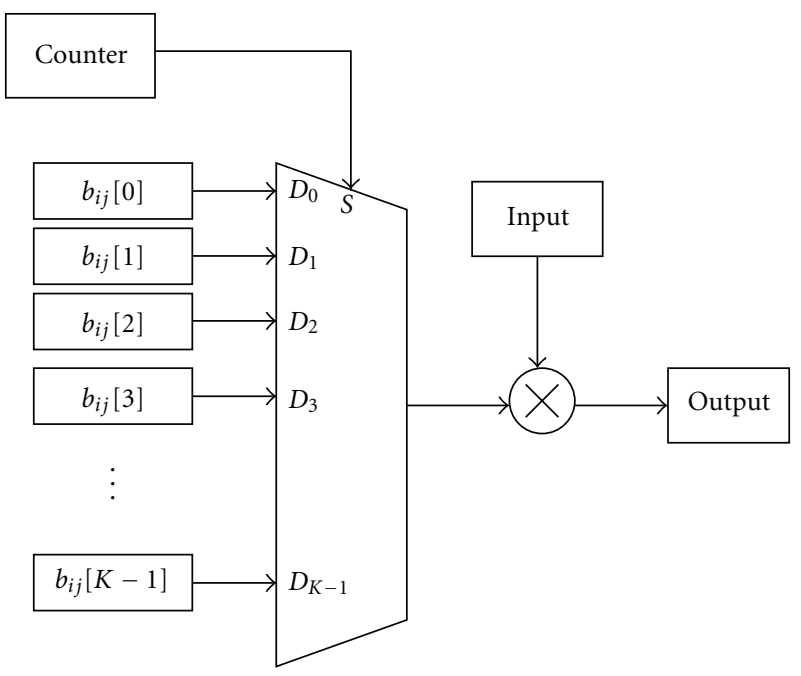

FIGURE 15: Fully multiplexed multiplier circuit for $\mathrm{K}$-time-multiplexed filters.

filters is shown in Figure 8. Relative error [85] in $\left|H\left(z_{1}, z_{2}\right)\right|$ is calculated as follows:

$$
\frac{\Delta H}{H}=\sum_{i=0}^{N-1} \sum_{j=0}^{N-1} \operatorname{Real}\left(S_{b_{i j}}^{\mid H\left(e^{\left.j \omega_{1}, e^{j \omega_{2}}\right) \mid}\right)} \frac{\Delta b_{i j}}{b_{i j}},\right.
$$

where $i+j \neq 0$. From Figure 8 for the usable frequency range $\omega_{2} \leq \pi / 2$, we observe that, for 2 nd-order beamformers, $W_{c}=15$-bit precision in the filter coefficients leads to beam accuracy within $3 \%$ which improves to better than $0.1 \%$ for $W_{c}=20$-bit precision. For 3rd-order beamformers, $W_{c}=$ 19 -bit precision in the filter coefficients leads to $3 \%$ accuracy, which improves to better than $0.1 \%$ accuracy when the filter coefficient precision is increased to $W_{c}=23$-bits.

3.5. Internal Register/Word Sizes. The word length of the signal at the feedback loop needs to be truncated. To maintain a minimum error, the size of the input signal has been decided based on the maximum value of the output signal. The design has been simulated for various word sizes and compared to the 64-bit precision Matlab outputs. For this experiment, we provided an impulsive UWB signal as an input to the filter because the operation and accuracy of the filter can be tested from the impulse response of the filter.

Tables 2 and 3 show the variation of quantization noise with the word size of the coefficients of the filter and the input size. In general, increased precision in the recursive spatio-temporal feedback sections leads to larger VLSI area and higher power consumption with lower speed due to larger CPDs. A compromise must be found depending on the needs of the target application that balances power, speed, accuracy, and chip area.

3.6. Look-Ahead Speed Optimization. The speed of the designs is maximized using fine-grain pipelining. Multilevel pipelining, that is, both inter-PPCM pipelining and intrapipelining, is used for maximum performance. 


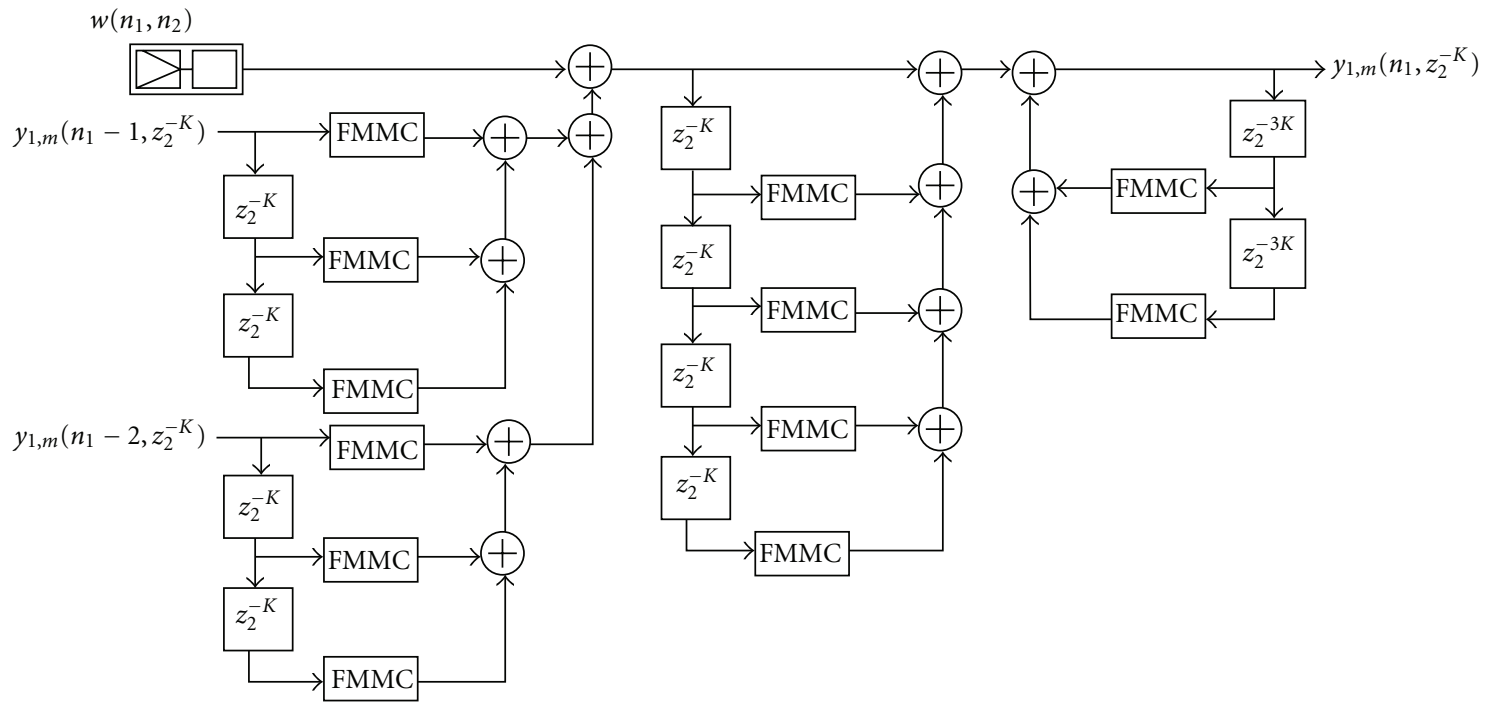

Figure 16: Time-multiplexed PPCM of 2nd-order beam filter bank with 3-stage scattered look-ahead pipelining for K-time-multiplexed beam filters.

TABLE 2: Calculation of error energy for unit impulse input for different output sizes of 2nd-order beam filter.

\begin{tabular}{lccccc}
\hline$(W, D)$ & \multicolumn{3}{c}{$\left(W_{c}, D_{c}\right)$} & $(17,-14)$ & $(16,-13)$ \\
\hline$(27,-17)$ & 0.0104 & $(19,-16)$ & $(18,-15)$ & 0.1107 & 4.0944 \\
$(26,-16)$ & 0.0321 & 0.0425 & 0.2292 & 0.1431 & 4.7827 \\
$(25,-15)$ & 0.1173 & 0.0831 & 0.2759 & 0.2335 & 5.1569 \\
$(24,-14)$ & 0.4520 & 0.2269 & 0.3645 & 0.7018 & 5.5859 \\
$(23,-13)$ & 1.6935 & 0.5424 & 0.6842 & 1.8941 & 6.3689 \\
$(22,-12)$ & 7.3510 & 2.1197 & 1.9373 & 7.7967 & 12.5538 \\
$(21,-11)$ & 25.4179 & 6.8815 & 7.1973 & 35.5036 & 3.8628 \\
\hline
\end{tabular}

TABLE 3: Calculation of unnormalized L2-error energy for unit impulse input for different output sizes of 3rd-order beam filter.

\begin{tabular}{cccccc}
\hline$(W, D)$ & \multicolumn{5}{c}{$\left(W_{c}, D_{c}\right)$} \\
& $(23,-19)$ & $(22,-18)$ & $(21,-17)$ & $(20,-16)$ & $(19,-15)$ \\
\hline$(34,-19)$ & 0.0496 & 0.1097 & 0.3805 & 12.7429 & 3.4765 \\
$(33,-18)$ & 0.0506 & 0.1148 & 0.3799 & 12.9468 & 3.4434 \\
$(32,-17)$ & 0.0544 & 0.1180 & 0.3922 & 12.4035 & 3.5761 \\
$(31,-16)$ & 0.0828 & 0.1381 & 0.4739 & 13.5182 & 3.4513 \\
$(30,-15)$ & 0.1786 & 0.2397 & 0.7910 & 14.0064 & 3.8871 \\
$(29,-14)$ & 0.5750 & 0.7209 & 1.6789 & 16.1490 & 4.6594 \\
\hline
\end{tabular}

Intrapipelining refers to internal optimization within the PPCMs. The feedback loop of an IIR filter inside the PPCM can be pipelined using look-ahead pipelining. Inter-PPCM pipelining refers to the pipelining of signal paths outside the PPCMs. The application of look-ahead pipelining to nonseparable 2D IIR digital filters was first proposed in [10]. Here, we pipeline each feedback loop of the proposed filters using stable scattered look-ahead pipelining (SLA) [11, 86, 87]. In SLA, additional zeros and poles which are at same angular distance as the original poles are introduced into the transfer function, enabling the $\mathrm{CPD}$ of the filter to be reduced.

If the denominator of the transfer function is in the form of $D(z)$,

$$
D(z)=\prod_{k=1}^{N}\left(1-p_{i} z^{-1}\right) .
$$

The general equation of $M$-stage SLA pipelining [86] is given by

$$
\begin{aligned}
H(z) & =\frac{N(z)}{D(z)} \\
& =\frac{N(z) \prod_{i=1}^{N} \prod_{k=1}^{M-1}\left(1-p_{i} e^{j 2 \pi k / M} z^{-1}\right)}{\prod_{i=1}^{N} \prod_{k=0}^{M-1}\left(1-p_{i} e^{j 2 \pi k / M} z^{-1}\right)} \\
& =\frac{N^{\prime}(z)}{D^{\prime}\left(z^{M}\right)} .
\end{aligned}
$$

The feedback loop for 2nd-order beam filter as shown in the following equation is pipelined for 3 stages in our design example:

$$
H_{1, m}\left(n_{1}, z_{2}\right)=\frac{1}{1+b_{01} z_{2}^{-1}+b_{02} z_{2}^{-2}} .
$$




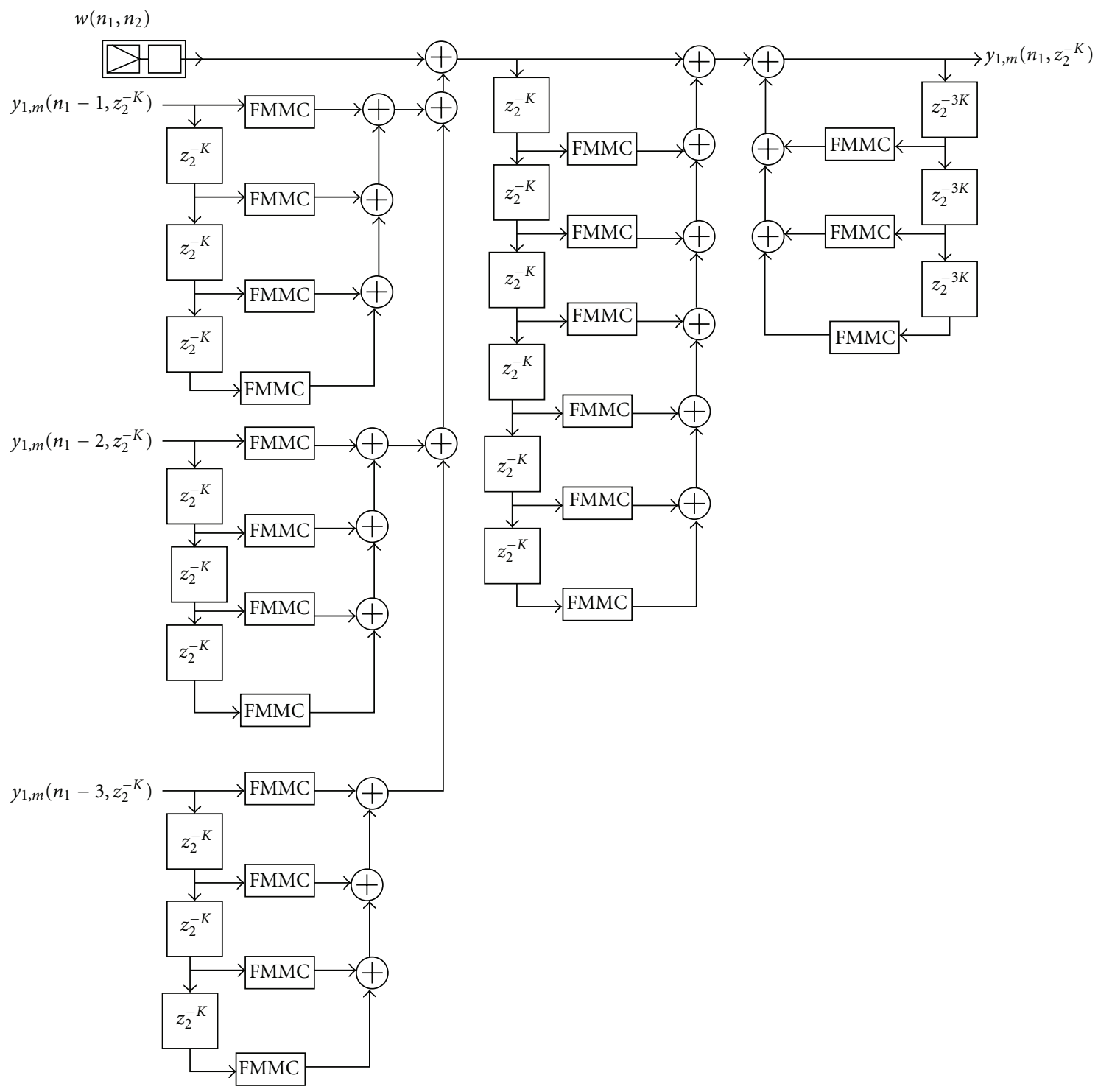

Figure 17: Time-multiplexed PPCM of 3rd-order beam filter bank with 3-stage scattered look-ahead pipelining for K-time-multiplexed beam filters.

Transfer function for 3-stage look-ahead pipelining of feedback loop is given by

$$
H_{1, m}^{\prime}\left(n_{1}, z_{2}\right)=\frac{1+A_{11} z_{2}^{-1}+A_{12} z_{2}^{-2}+A_{13} z_{2}^{-3}+A_{14} z_{2}^{-4}}{1+B_{11} z_{2}^{-3}+B_{12} z_{2}^{-6}}
$$

where

$$
\begin{aligned}
& A_{11}=-b_{01}, \\
& A_{12}=\left(b_{01}^{2}-b_{02}\right), \\
& A_{13}=-b_{01} b_{02}, \\
& A_{14}=b_{02}^{2}, \\
& B_{11}=-\left(-b_{01}^{3}+3 b_{01} b_{02}\right), \\
& B_{12}=b_{02}^{3} .
\end{aligned}
$$

For the 3rd order, the feedback loop is described using the following equation:

$$
H_{1, m}\left(n_{1}, z_{2}\right)=\frac{1}{1+b_{01} z_{2}^{-1}+b_{02} z_{2}^{-2}+b_{03} z_{2}^{-3}} .
$$

The transfer function for 3-stage look-ahead pipelining of feedback loop is given by

$$
\begin{aligned}
H_{1, m}^{\prime}\left(n_{1}, z_{2}\right)= & \frac{\left(1+A_{11} z_{2}^{-1}+A_{12} z_{2}^{-2}+A_{13} z_{2}^{-3}\right)}{\left(1+B_{11} z_{2}^{-3}+B_{12} z_{2}^{-6}+B_{13} z_{2}^{-9}\right)} \\
& +\frac{\left(A_{14} z_{2}^{-4}+A_{15} z_{2}^{-5}+A_{16} z_{2}^{-6}\right)}{\left(1+B_{11} z_{2}^{-3}+B_{12} z_{2}^{-6}+B_{13} z_{2}^{-9}\right)},
\end{aligned}
$$




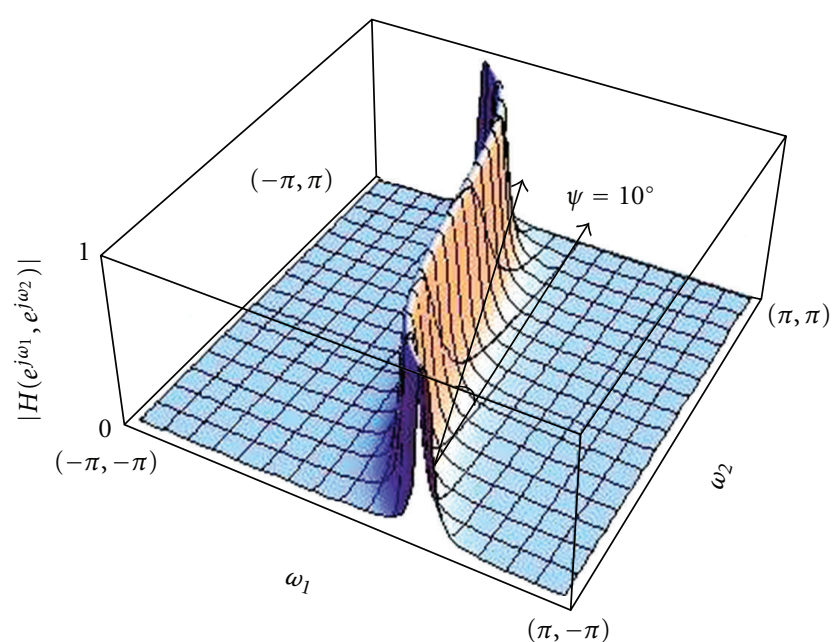

FIGURE 18: Magnitude frequency response of 2D 2nd-order beam filter for impulse input signal.

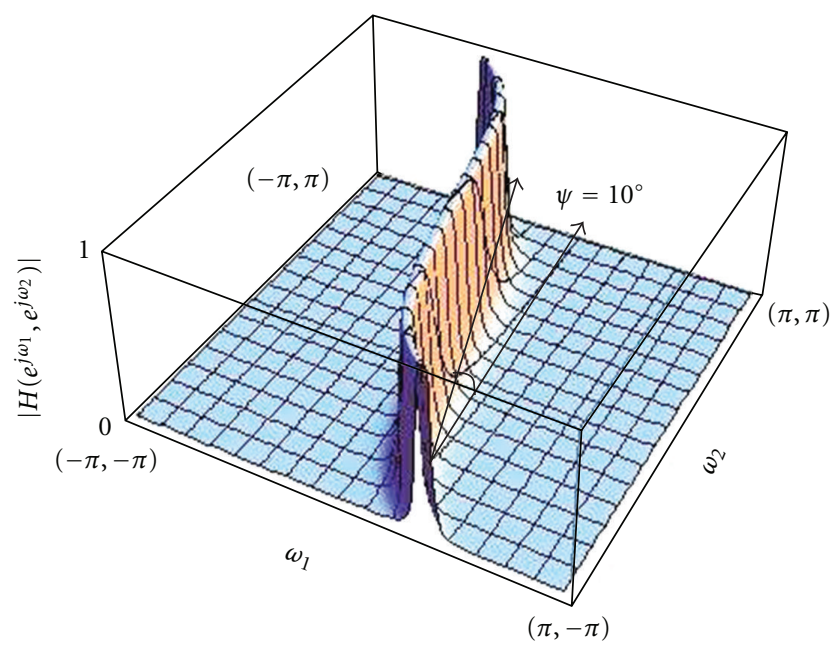

FIGURE 19: Magnitude frequency response of 2D 3nd-order beam filter for impulse input signal.

where

$$
\begin{aligned}
& A_{11}=-b_{01}, \\
& A_{12}=\left(b_{01}^{2}-b_{02}\right), \\
& A_{13}=2 b_{03}-b_{01} b_{02}, \\
& A_{14}=b_{02}^{2}-b_{02} b_{03}, \\
& A_{15}=-b_{02} b_{03}, \\
& A_{16}=b_{03}^{2}, \\
& B_{11}=b_{01}^{3}-3 b_{01} b_{02}+3 b_{03}, \\
& B_{12}=b_{02}^{3}+2 b_{01} b_{03}+3 b_{03}^{2}, \\
& B_{13}=b_{03}^{3} .
\end{aligned}
$$

Figure 9 shows the realization of $H_{2}\left(z_{1}\right)$ of 2 nd-order beam filter. $H_{2}\left(z_{1}\right)$ is a spatial function. Hence, it is realized as sum of three consecutive 1D outputs given by the PPCMs of $H_{1}\left(z_{1}, z_{2}\right) . H_{3}\left(z_{2}\right)$ is temporal function and it is realized as consecutive 1D filters as shown in Figure 10. Similar are the realizations for the 3rd-order filter, as shown in Figure 11 and described by Figure 12 .

3.7. Time Multiplexing for Folded Architectures. Timemultiplexed systolic-array designs are useful for the design of fan filters [88] whose transfer function is given in the following. Figure 13 shows how fan filters are designed using time-multiplexed filters and are described using [12]

$$
H_{\text {Fan }}\left(z_{1}, z_{2}\right)=\sum_{k=1}^{K} H_{\text {beam }, k}\left(z_{1}, z_{2}\right) T_{\mathrm{FIR}, k}\left(z_{2}\right),
$$

where $T_{\mathrm{FIR}, k}\left(z_{2}\right)$ are subbands of a perfect reconstruction FIR bandpass filter bank.

Time multiplexing of $K$-filters needs the inputs to be upsampled by $K \in Z^{+}$with copying $[88,89]$. The input signal consists of $K$ samples pertaining to each original sample and are passed through the time-multiplexed filter. In Figure 14, we provide an overview of signal flow in timemultiplexed design of the $K$ beam filters. These filters give $K$ outputs which are to be demultiplexed before being applied to the fan filter bank perfect reconstruction FIR bandpass filters [12]. The time multiplexing of the folded architecture caused each unit delay to be increased to

$$
\Delta T=K \Delta T_{\mathrm{clk}}
$$

where $K$ is the number of inputs ( $K=4$, in the example provided) and $\Delta T_{\text {clk }}$ is the clock delay.

The signal flow inside the time-multiplexed PPCM is such that the coefficients of feedback terms of $K$ filters are given to a two-input multiplier through a commutating multiplexer. Input signal $w\left(\Delta x n_{1}, c \Delta T_{s} n_{2}\right)$ is given to the other input of multiplier. A counter is used to select feedback coefficient given to multiplexer. We have designed the filter bank with four filters. Hence, the counter runs from 0 to 3 and restarts. Therefore, when there exists $K$ filter coefficients, the required counter must continuously count up 0 to $\log _{2} K$. The critical-path delay reduces as the architecture is finegrain pipelined with SLA pipelining for the feedback path. Figure 15 shows each fully multiplexed multiplier circuit (FMMC) design for beam filters. 2nd-order and 3rd-order time-multiplexed designs are as shown in Figures 16 and 17, respectively, with $\Delta T$ as in (22).

\section{Simulation and Implementation}

4.1. The 2D Frequency Response. The obtained 2D magnitude frequency response of a 2nd-order frequency-planar beam plane-wave filter matches the ideal frequency response shown in Figure 18. Figure 19 shows the magnitude frequency response of 3rd-order plane-wave filter. We observe unavoidable warping effect in discrete domain systems due to the use of the $2 \mathrm{D}$ bilinear transform. Warping effects can 


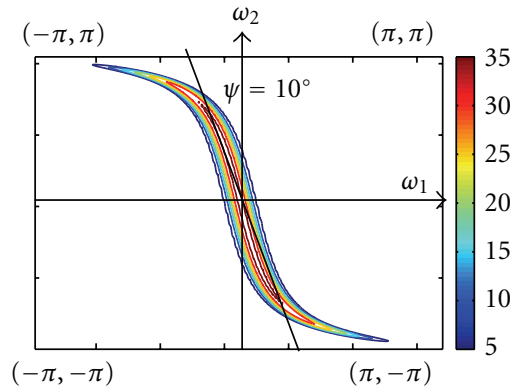

(a)

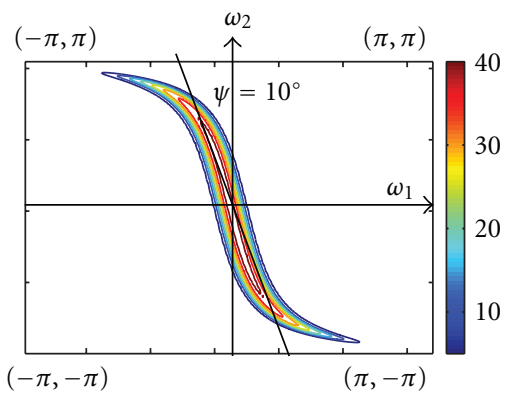

(d)

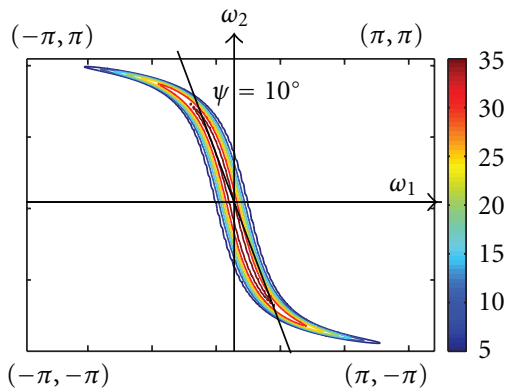

(b)

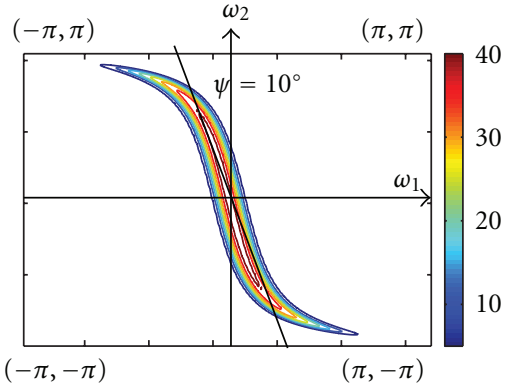

(e)

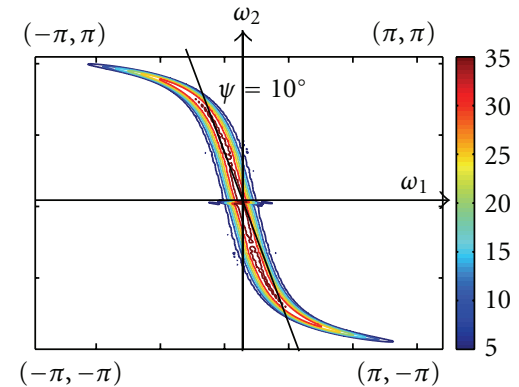

(c)

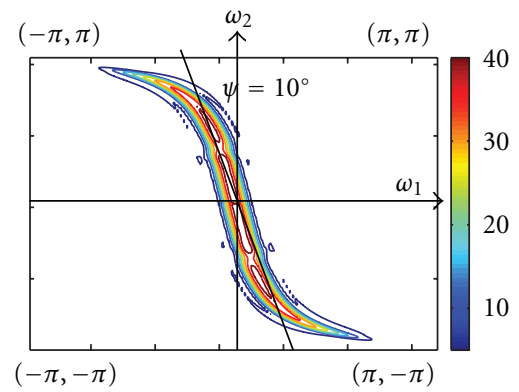

(f)

Figure 20: Contour plots of beam filter response in log scale for (a) ideal 2nd-order beam filter, (b) 2nd-order beam filter for $W=27$, (c) 2nd-order beam filter for $W=21$, (d) ideal 3rd-order beam filter, (e) 3rd-order beam filter for $W=34$, (f) 3rd-order beam filter for $W=29$.

be avoided by temporally oversampling the signal such that the spectrum lies totally within the straight-line region of the beam response. Figure 20 shows contours of the magnitude response of the filters in log scale. Plots for infinite precision, highest fixed-point precision, as well as lowest fixed-point precision are provided. It can be seen that the transition (rolloff) of the 3rd-order plane-wave filter is sharper compared to that of 2 nd-order filter. The performance of the designed finite precision digital systolic-array circuit is measured by calculating the $L 2$ error energy due under quantization effects. The output obtained from difference equation (from Matlab, at 64-bit precision) is considered as ideal output, and the error is calculated by the difference of ideal output and the fixed-point measurement from the FPGA device. The $L 2$ energy of the error, $E_{\text {error }}$, is calculated as follows:

$$
E_{\text {error }}=\sum_{n_{2}=1}^{N_{2}} \sum_{n_{1}=1}^{N_{1}}|| y_{\text {ideal }}^{\prime}\left(e^{j \omega_{1}}, e^{j \omega_{2}}\right)|-| y^{\prime}\left(e^{j \omega_{1}}, e^{j \omega_{2}}\right)||^{2}
$$

where $y_{\text {ideal }}^{\prime}\left(e^{j \omega_{1}}, e^{j \omega_{2}}\right)$ is the Fast Fourier Transform of the ideal output obtained using difference equation from Matlab and $y^{\prime}\left(e^{j \omega_{1}}, e^{j \omega_{2}}\right)$ is the Fast Fourier Transform of the output obtained from the hardware design. We have tabulated unnormalized L2-error energies as a metric for quantization noise levels for different values of word sizes for 2nd-order and 3rd-order plane-wave filters in Tables 2 and 3, respectively.
4.2. Broadband Signal Filtering. We demonstrate the directional selectivity of the proposed frequency-planar planewave beam filters by employing a Gaussian impulsive ultrawideband signal [90] as input to the filter. The input to the filters that is shown in Figure 21(a) is a combination of three signals with space-time DOAs $-40^{\circ}, 10^{\circ}$, and $41^{\circ}$ given by

$$
w\left(n_{1}, n_{2}\right)=\cos \left(\omega_{k} l_{k}\right) e^{-\lambda_{k} l_{k}^{2}}
$$

where $l_{k}=n_{1} \sin \left(\theta_{k}\right)+n_{2}, k=0,1,2$. We used $\omega_{k}=1.12$, $\lambda_{k}=0.1, \theta_{0}, \theta_{1}, \theta_{2}$ such that they have space-time DOA, $\psi_{0}=-40^{\circ}, \psi_{1}=10^{\circ}, \psi_{2}=41^{\circ}$. The 2 nd-order beam filter selectively filters signal with space-time DOA of $10^{\circ}$ and attenuates the signal (pulse peak) with $\psi_{0}=-40^{\circ}$ by $40.11 \mathrm{~dB}$ and signal (pulse peak) with $\psi_{2}=41^{\circ}$ by $37.22 \mathrm{~dB}$. Similarly, 3rd-order beam filter selects signal with space-time DOA of $10^{\circ}$ and attenuates the signal (pulse peak) with $\psi_{0}=$ $-40^{\circ}$ by $50.305 \mathrm{~dB}$ and signal (pulse peak) with $\psi_{2}=41^{\circ}$ by $52.2 \mathrm{~dB}$.

The directional attenuation of the signals was calculated using $20 \log _{10}|A| \mathrm{dB}$ where $A$ is the magnitude of the attenuated signal. Attenuation in signal energy was calculated by correlation. The directional enhancement of the energy of desired signal (or attenuation of the undesired signal) was calculated using $10 \log _{10}|A| \mathrm{dB}$. The attenuation in energy of the signal with $\psi_{0}=-40^{\circ}$ is $18.78 \mathrm{~dB}$ for 2 nd-order plane-wave filter and $26.2 \mathrm{~dB}$ for $3 \mathrm{rd}$-order plane-wave filter. Energy of the signal with $\psi_{2}=41^{\circ}$ is attenuated by 


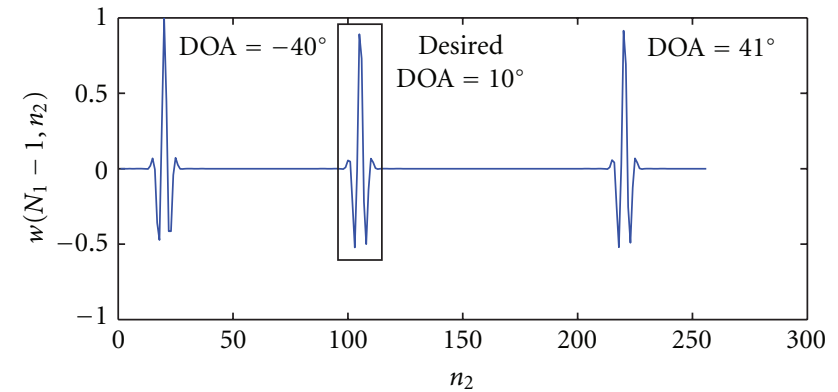

(a) Input signal of three 2D Gaussian pulse functions at $N_{1}=31$ with combination of three different space-time DOA signals

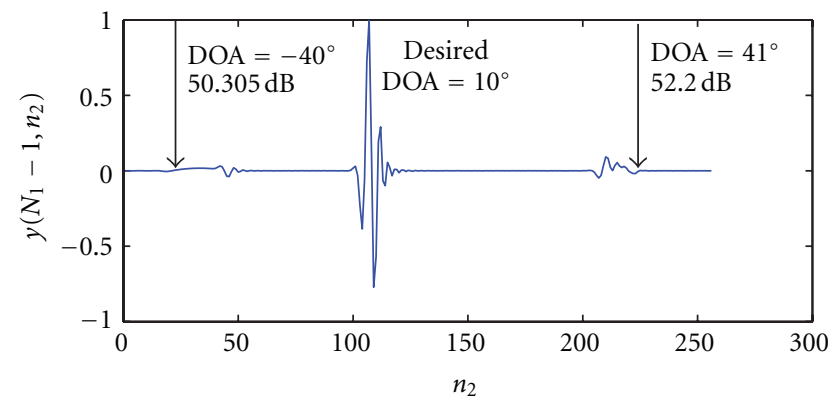

(c) Output signal of 2D 3rd-order filter at $N_{1}=31$

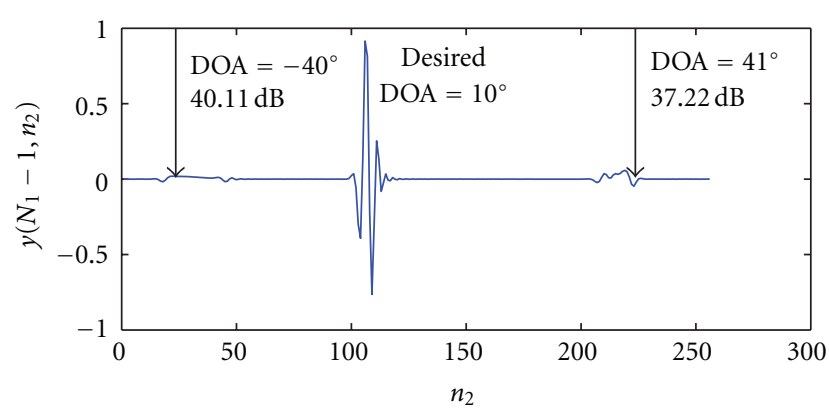

(b) Output signal of 2D 2nd-order filter at $N_{1}=31$

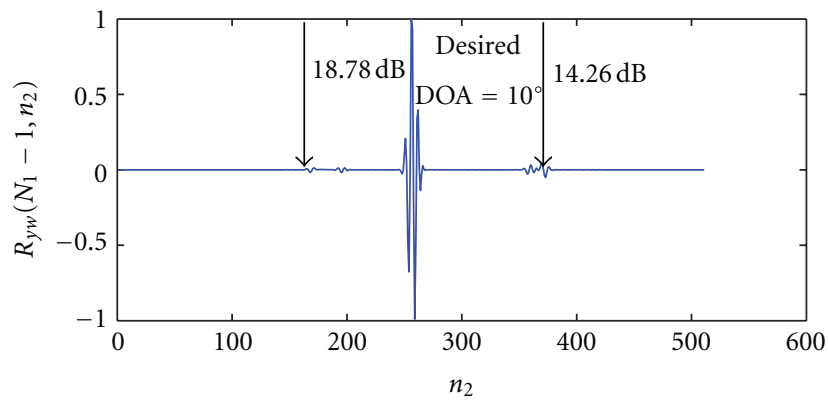

(d) Cross-correlated signal of input and output signal at $N_{1}=31$ for 2ndorder filter

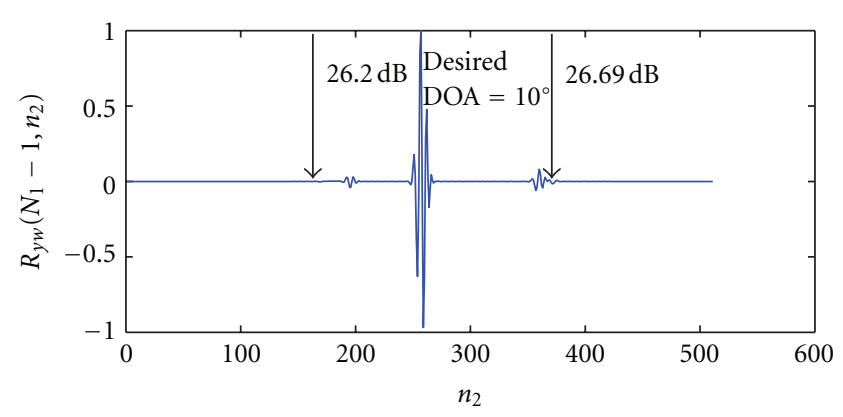

(e) Cross-correlated signal of input and output signal at $N_{1}=31$ for 3rdorder filter

Figure 21: Input and output Gaussian signals in time domain.

$14.26 \mathrm{~dB}$ by 2 nd-order plane-wave filter and $26.69 \mathrm{~dB}$ by $3 \mathrm{rd}-$ order plane-wave filter. We observe that the attenuation of the undesired signals improves for the higher-order planewave filter, which confirms that the directional selectivity of a beamformer improves with the order of the filter. Figure 22(a) shows the 2D spectrum of the input Gaussian broadband signal. In Figure 22(b), we show the output of the 2nd-order beam filter, followed by Figure 22(c), which shows the output of 3rd-order beam filter for broadband Gaussian modulated cosine waves.

4.3. Beam Patterns (Array Factor). Directional enhancement of plane-wave beam filters can be observed through beam patterns of filter at different frequencies. Figure 23 shows comparison between beam patterns of 1st-order filter, 2ndorder filter, and 3 rd-order filter for frequencies $\pi / 4, \pi / 2,2 \pi / 3$ radians, respectively. The polar plot of $N$ th-order filter can be obtained using magnitude of the transfer functions which is given by

$$
H\left(z_{1}, z_{2}\right)=\frac{\left(1+z_{1}^{-1}\right)^{N}\left(1+z_{2}^{-1}\right)^{N}}{1+\sum_{i=0}^{N} \sum_{j=0}^{N} b_{i j} z_{1}^{-i} z_{2}^{-j}},
$$

where $i+j \neq 0$ for feedback loop

$$
\begin{gathered}
z_{1}=e^{j \omega_{1}}, \\
z_{2}=e^{j \omega_{2}}, \\
\omega_{1}=-\omega_{2} \sin \psi .
\end{gathered}
$$

By substituting $\omega_{1}, z_{1}$, and $z_{2}$ in terms of $\omega_{2}, H\left(z_{1}, z_{2}\right)$ is made dependent on $\omega_{2}$. The magnitude of normalized function $H\left(z_{1}, z_{2}\right)$ is calculated by

$$
P\left(\psi, \omega_{2}\right)=\frac{\left|H\left(e^{j \omega_{2} \sin \psi}, e^{j \omega_{2}}\right)\right|}{\max \left(\left|H\left(e^{j \omega_{2} \sin \psi}, e^{j \omega_{2}}\right)\right|\right)} .
$$



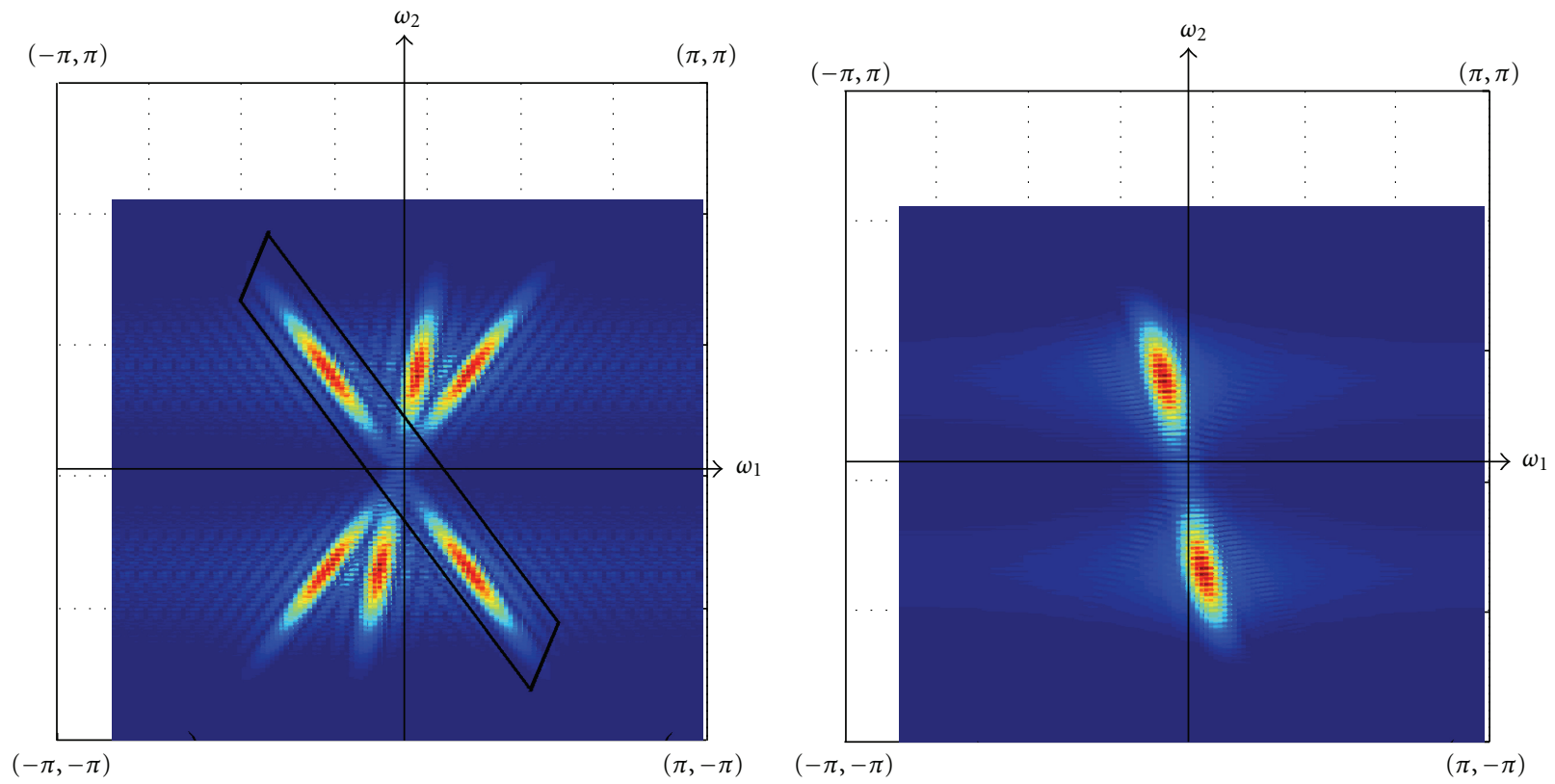

(a) $X-Y$ view of three 2D Gaussian pulse functions in frequency domain

(b) $X-Y$ view of frequency response of 2D 2nd-order filter for Gaussian pulse signals

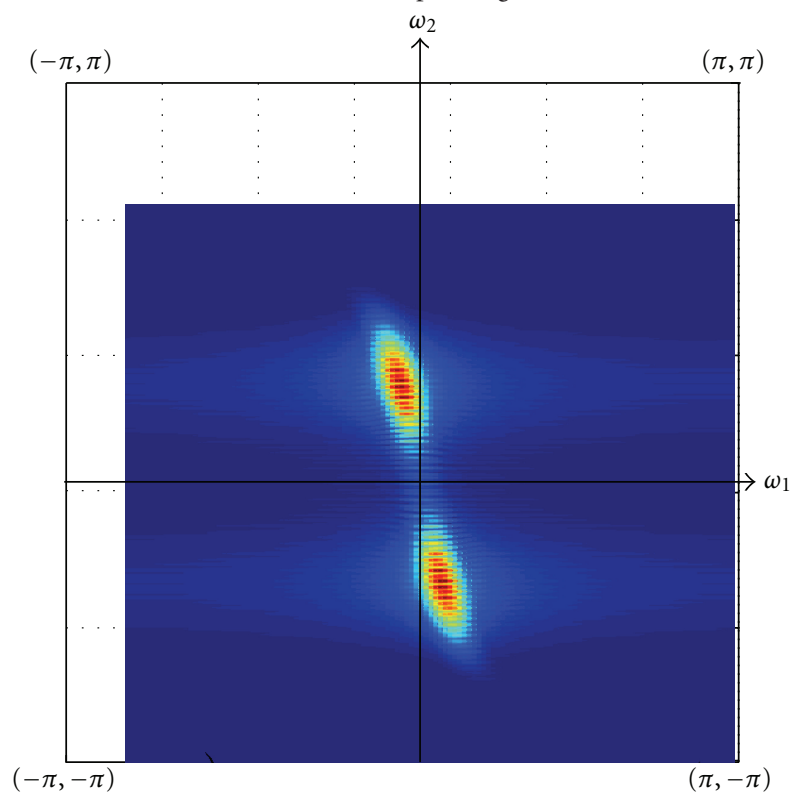

(c) $X-Y$ view of frequency response of 2D 3rd-order filter for Gaussian pulse signals

FIGURE 22: Input and output Gaussian signals in frequency domain.

The values of $b_{i j}$ for $N$ th-order function are same as the values considered for filter design. The plots shown in Figure 23 give the polar plot (in $\mathrm{dB}$ scale) of the functions $H\left(z_{1}, z_{2}\right)$ using $\log$ scale which means the magnitude of the function is calculated as $20 \log _{10}\left|H\left(z_{1}, z_{2}\right)\right|$.

4.4. FPGA-Based ASIC Emulation. We employ FPGA-based logic emulation for low-cost prototyping of potential RF digital application specific integrated circuits (ASICs). The proposed designs of 2 nd-order and 3rd-order plane-wave beam filters are implemented on a Xilinx Virtex 6 xc6vsx475t$2 \mathrm{ff} 1759$ device for different fixed-point precision levels. The 3 rd-order plane-wave beam filter is more complicated compared to 2nd-order filter due to higher multiplier and adder complexity. To ensure place-and-route success, we limit the number of PPCMs to 10 for each case. Implementation was first simulated and then targeted to a Virtex 6 xc6vsx475t2 ff1759 FPGA board. We optimized the real-time clock speed of the proposed designs by applying 3-stage SLA pipelining. These filters were implemented both with and without SLA 


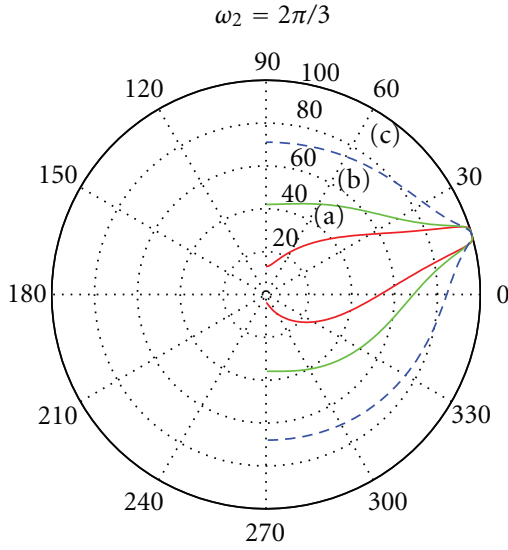

(a)

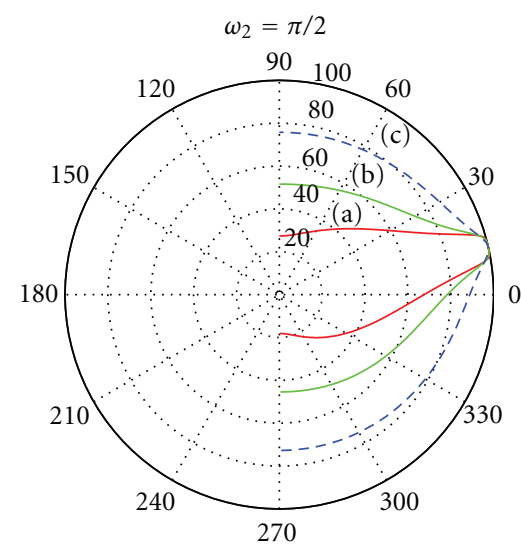

(b)

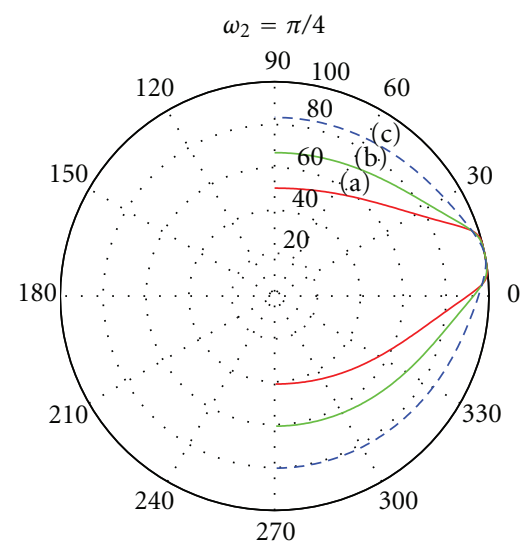

(c)

FIGURE 23: Beam patterns of 2D filters of (a) 3rd-order, (b) 2nd-order, (c) 1st-order along space-time DOA, $\psi=10^{\circ}$ for $\omega_{2}=2 \pi / 3, \omega_{2}=\pi / 2$, $\omega_{2}=\pi / 4$ in order.

TABLE 4: Hardware resource consumptions for pipelined 2ndorder beam filter with 3-stage SLA for different word lengths for 20 PPCMs.

\begin{tabular}{lccccc}
\hline \multirow{2}{*}{$W, D)$} & $T_{\mathrm{CPD}}$ & $F_{\max }$ & \multicolumn{3}{c}{ FPGA source consumptions } \\
& $(\mathrm{ns})$ & $(\mathrm{MHz})$ & Slice Reg.s & LUTs & FFs \\
\hline$(27,-17)$ & 9.220 & 106.757 & 54,235 & 188,387 & 54,215 \\
$(26,-16)$ & 8.945 & 108.968 & 54,048 & 179,186 & 53,969 \\
$(25,-15)$ & 8.749 & 111.271 & 51,926 & 165,477 & 51,776 \\
$(24,-14)$ & 8.654 & 113.960 & 47,707 & 155,265 & 47,707 \\
$(23,-13)$ & 8.442 & 116.536 & 41,103 & 120,694 & 41,065 \\
$(22,-12)$ & 8.297 & 119.360 & 41,103 & 120,431 & 41,065 \\
\hline
\end{tabular}

TABLE 5: Hardware resource consumptions for pipelined 2nd-order beam filter without LA for different word lengths for 20 PPCMs.

\begin{tabular}{lccccc}
\hline \multirow{2}{*}{$(W, D)$} & $T_{\mathrm{CPD}}$ & $F_{\max }$ & \multicolumn{3}{c}{ FPGA source consumptions } \\
& $(\mathrm{ns})$ & $(\mathrm{MHz})$ & Slice Reg.s & LUTs & FFs \\
\hline$(27,-17)$ & 10.390 & 94.099 & 36,098 & 107,604 & 36,078 \\
$(26,-16)$ & 10.069 & 98.260 & 36,502 & 105,162 & 36,482 \\
$(25 .-15)$ & 9.825 & 99.482 & 35,010 & 97,111 & 34,860 \\
$(24,-14)$ & 9.766 & 99.900 & 31,648 & 92,578 & 31,610 \\
$(23,-13)$ & 9.695 & 102.048 & 28,590 & 78,706 & 28,570 \\
$(22,-12)$ & 9.656 & 102.222 & 26,578 & 72,774 & 26,558 \\
\hline
\end{tabular}

in order to observe the relative improvement in performance. Tables 4, 5, 6, and 7 show the FPGA hardware resources such as slice registers, look-up tables (LUTs), flip-flops (FFs), $\mathrm{CPD}$, and maximum clock frequency. We observe a reduction in speed of operation of the 3rd-order plane-wave filter due to increased complexity at same level of pipelining as 2nd-order plae-wave filter. Time-multiplexed filters having corresponding folded digital architectures were physically implemented on the same board, and resource consumptions are tabulated in Tables 8 and 9, respectively. Folding and time multiplexing were applied to both designs having the SLA pipelining. At this stage, we use FPGA prototypes for
TABLE 6: Hardware resource consumptions for pipelined 3rd-order beam filter with SLA for different word lengths for 10 PPCMs.

\begin{tabular}{cccccc}
\hline \multirow{2}{*}{$(W, D)$} & $T_{\mathrm{CPD}}$ & $F_{\max }$ & \multicolumn{3}{c}{ FPGA source consumptions } \\
& $(\mathrm{ns})$ & $(\mathrm{MHz})$ & Slice Reg.s & LUTs & FFs \\
\hline$(34,-19)$ & 12.703 & 76.417 & 62,330 & 209,945 & 62,263 \\
$(33,-18)$ & 11.717 & 83.458 & 60,435 & 192,506 & 60,327 \\
$(32,-17)$ & 11.048 & 89.365 & 58,581 & 182,595 & 58,509 \\
$(31,-16)$ & 10.751 & 90.9421 & 57,761 & 175,520 & 57,268 \\
$(30,-15)$ & 9.711 & 101.389 & 54,833 & 157,486 & 54,479 \\
$(29,-14)$ & 9.593 & 103.274 & 52,931 & 146,335 & 52,490 \\
\hline
\end{tabular}

verification of operation at an order of magnitude lower clock frequency than the final expected RF digital realization.

4.5. Computational Complexity. Table 10 shows comparison of computational complexity and throughput for one PPCM between 2nd-order and 3rd-order beam filters for different stages of SLA. VLSI metrics area time $\left(A T^{2 n}, 0 \leq n \leq 1\right)$ [91] is calculated as a measurement of complexity to find the main constraints in designing the filters. In VLSI systems, $n=1$ is used for cases where low chip area is more important than clock speed. Similarly, $n=2$ is used for cases where clock speed is the driving factor. Table 11 gives measures of both $A T$ and $A T^{2}$ for 2nd-order pipelined designs, both with and without LA, for different levels of fixed-point precision. Furthermore, Table 12 gives the same metrics for the 3rdorder filter architecture.

\section{Conclusion}

Highly directional steerable ultra-wideband digital antenna aperture arrays have useful applications in wireless communications, radar, radio astronomy, spectrum sensing, RF imaging and remote sensing. We propose novel massivelyparallel systolic-array architectures for the real-time digital realization of beamforming 2D IIR frequency-planar beam plane-wave filters based on resistively terminated LC ladder 
TABLE 7: Hardware resource consumptions for pipelined 3nd-order beam filter without LA for different word lengths for 10 PPCMs.

\begin{tabular}{lccccc}
\hline \multirow{2}{*}{$(W, D)$} & $T_{\mathrm{CPD}}$ & $F_{\max }$ & \multicolumn{3}{c}{ FPGA source consumptions } \\
& $(\mathrm{ns})$ & $(\mathrm{MHz})$ & Slice Reg.s & LUTs & FFs \\
\hline$(34,-19)$ & 12.983 & 75.506 & 51,546 & 107,959 & 51,510 \\
$(33,-18)$ & 12.977 & 76.207 & 50,747 & 101,496 & 50,666 \\
$(32,-17)$ & 12.939 & 76.262 & 48,166 & 96,815 & 48,157 \\
$(31,-16)$ & 11.172 & 87.777 & 63,434 & 86,993 & 63,416 \\
$(30,-15)$ & 11.012 & 89.039 & 39,937 & 85,058 & 39,919 \\
$(29,-14)$ & 10.843 & 91.082 & 39,255 & 79,450 & 39,172 \\
\hline
\end{tabular}

TABLE 8: Hardware resource consumptions for time-multiplexed 2nd-order beam filter for different word lengths for 10 PPCMs.

\begin{tabular}{lccccc}
\hline \multirow{2}{*}{$(W, D)$} & $T_{\mathrm{CPD}}$ & $F_{\max }$ & \multicolumn{3}{c}{ FPGA source consumptions } \\
& $(\mathrm{ns})$ & $(\mathrm{MHz})$ & Slice Reg.s & LUTs & FFs \\
\hline$(27,-17)$ & 16.210 & 60.55 & 27,497 & 169,646 & 27,159 \\
$(26,-16)$ & 15.925 & 62.38 & 26,717 & 160,303 & 26,035 \\
$(25,-15)$ & 15.627 & 63.5 & 25,055 & 149,922 & 24,903 \\
$(24,-14)$ & 15.363 & 64.4 & 24,137 & 137,879 & 23,803 \\
$(23,-13)$ & 14.056 & 69.9 & 23,066 & 136,398 & 22,818 \\
$(22,-12)$ & 13.747 & 71.8 & 22,276 & 125,381 & 21,686 \\
\hline
\end{tabular}

TABle 9: Hardware resource consumptions for time-multiplexed 3rd-order beam filter for different word lengths for 6 PPCMs.

\begin{tabular}{lccccc}
\hline \multirow{2}{*}{$(W, D)$} & $T_{\mathrm{CPD}}$ & $F_{\max }$ & \multicolumn{3}{c}{ FPGA source consumptions } \\
& $(\mathrm{ns})$ & $(\mathrm{MHz})$ & Slice Reg.s & LUTs & FFs \\
\hline$(34,-19)$ & 25.897 & 38.77 & 19,299 & 195,257 & 18,690 \\
$(33,-18)$ & 25.438 & 39.24 & 19,250 & 187,678 & 18,054 \\
$(32,-17)$ & 24.487 & 40.17 & 18,217 & 173,988 & 17,461 \\
$(31,-16)$ & 24.477 & 40.82 & 17,670 & 160,669 & 16,808 \\
$(30,-15)$ & 23.315 & 42.56 & 17,067 & 152,211 & 16,203 \\
$(29,-14)$ & 23.065 & 43.51 & 16,689 & 141,785 & 15,610 \\
\hline
\end{tabular}

TABLE 10: Computational complexity and throughput of 2nd-order and 3rd-order beam filters of N PPCMs.

\begin{tabular}{cccccc}
\hline$N$ & $\begin{array}{c}\text { Order of } \\
\text { SLA }\end{array}$ & $\begin{array}{c}\text { Mults. } \\
\text { per PPCM }\end{array}$ & $\begin{array}{c}\text { Mult.s } \\
\text { per sec }\end{array}$ & $\begin{array}{c}\text { Adders } \\
\text { per PPCM }\end{array}$ & $\begin{array}{c}\text { Add./sub.s } \\
\text { per sec }\end{array}$ \\
\hline 2 & None & 8 & $8 N F_{\text {clk }}$ & 8 & $8 N F_{\text {clk }}$ \\
3 & None & 15 & $15 N F_{\text {clk }}$ & 15 & $15 N F_{\text {clk }}$ \\
2 & 2 & 10 & $10 N F_{\text {clk }}$ & 10 & $10 N F_{\text {clk }}$ \\
3 & 2 & 18 & $18 N F_{\text {clk }}$ & 18 & $18 N F_{\text {clk }}$ \\
2 & 3 & 12 & $12 N F_{\text {clk }}$ & 12 & $12 N F_{\text {clk }}$ \\
3 & 3 & 21 & $21 N F_{\text {clk }}$ & 21 & $21 N F_{\text {clk }}$ \\
\hline
\end{tabular}

networks. The proposed architectures are aimed at 2ndand 3rd-order beam filters and are based on the recently proposed 1st-order beam filter architecture [10]. These beamformers offer both low computational complexity and UWB performance.

The proposed architectures enable RF throughputs when eventually realized using high-speed CMOS technology. The proposed architectures are evaluated for correct operation,
TABLE 11: Area time constraints for 2nd-order filter.

\begin{tabular}{ccccc}
\hline & \multicolumn{2}{c}{ Without LA } & \multicolumn{2}{c}{ With SLA } \\
$W$ & $A T$ & $A T^{2}$ & $A T$ & $A T^{2}$ \\
& {$[L]^{2}[T] \times$} & {$[L]^{2}[T]^{2} \times$} & {$[L]^{2}[T] \times$} & {$[L]^{2}[T]^{2} \times$} \\
& $10^{-12}$ & $10^{-21}$ & $10^{-12}$ & $10^{-21}$ \\
\hline 27 & 1118.1 & 11616.1 & 1736.9 & 16014.5 \\
26 & 1058.9 & 10661.8 & 1602.8 & 14337.2 \\
25 & 954.1 & 9374.2 & 1447.8 & 12666.4 \\
24 & 904.1 & 8829.6 & 1343.7 & 11628.1 \\
23 & 763.1 & 7397.8 & 1018.9 & 8601.5 \\
22 & 702.7 & 6785.3 & 999.2 & 8290.5 \\
\hline
\end{tabular}

TABLe 12: Area time constraints for 3rd-order filter.

\begin{tabular}{ccccc}
\hline & \multicolumn{2}{c}{ Without LA } & \multicolumn{2}{c}{ With SLA } \\
$W$ & $A T$ & $A T^{2}$ & $A T$ & $A T^{2}$ \\
& {$[L]^{2}[T] \times$} & {$[L]^{2}[T]^{2} \times$} & {$[L]^{2}[T] \times$} & {$[L]^{2}[T]^{2} \times$} \\
& $10^{-12}$ & $10^{-21}$ & $10^{-12}$ & $10^{-21}$ \\
\hline 34 & 1401.6 & 18197.4 & 2666.9 & 33878.0 \\
33 & 1317.1 & 17092.2 & 2255.6 & 26428.8 \\
32 & 1252.7 & 16208.5 & 2017.3 & 22287.2 \\
31 & 971.9 & 10857.9 & 1887.0 & 20287.3 \\
30 & 936.6 & 10314.5 & 1529.3 & 14851.4 \\
29 & 861.5 & 9341.0 & 1403.7 & 13466.5 \\
\hline
\end{tabular}

area, time, and complexity metrics as well as beam sensitivity and noise levels as a function of finite precision. Extensive prototype FPGA realizations, simulations, and FPGA-based emulations of beamforming performance are used here to validate the architectures and design procedures for high-performance digital UWB beamforming applications. As part of the proposed optimized designs, a fine-grain pipelined systolic-array 2D IIR plane-wave beam filters of orders 2 and 3 have been designed and optimized using scattered look-ahead to reduce the CPD of the digital circuits. Low CPD results in high real-time throughput with corresponding increase in clock frequency. The architectures were evaluated using $\mathrm{CPD}(T)$, area $(A)$, as well as $A T$ and $A T^{2}$.

The proposed 2nd-order design filter architecture is verified using a realistic Gaussian modulated cosine wave input signal consisting of several plane-waves. The filter is designed to enhance the plane-wave having space-time $\mathrm{DOA}=10^{\circ}$, while attenuating the undesired signal up to $40.11 \mathrm{~dB}$ for space-time $\mathrm{DOA}=-40^{\circ}$ and $37.22 \mathrm{~dB}$ for space-time DOA $=41^{\circ}$. Similarly, the 3rd-order filter provided up to $50.305 \mathrm{~dB}$ of stopband rejection for space-time $\mathrm{DOA}=-40^{\circ}$ and $52.2 \mathrm{~dB}$ of stop-band rejection for spacetime $\mathrm{DOA}=41^{\circ}$ for a broadband Gaussian-modulated cosine-wave signal. The corresponding hardware architectures were verified on FPGA chip using measured unit impulse responses obtained using stepped FPGA hardware cosimulation. The $2 \mathrm{D}$ frequency response of the measured impulse response from the FPGA chip is compared with a reference response obtained from the difference equation by calculating the $L 2$ error energy for different finite precision sizes. 
Furthermore, the proposed architectures were folded and time-multiplexed to form a filter bank. The timemultiplexed filters have also been physically implemented on Xilinx Virtex6 xc6vsx475t-2ff1759 FPGA board. Future work involves digital CMOS integrated circuits using ASIC standard cells for RF clock frequencies with applications in UWB real-time electromagnetic beamforming antenna arrays.

\section{References}

[1] M. Ghavami and R. Kohno, "Frequency selective broadband beamforming using 2D digital filters," in Proceedings of the IEEE 51st Vehicular Technology Conference (VTC '00), vol. 3, pp. 2522-2526, May 2000.

[2] L. T. Bruton and N. R. Bartley, "Three-dimensional Image Processing using the Concept of Network Resonance," IEEE Transactions on Circuits and Systems, vol. 32, no. 7, pp. 664672, 1985.

[3] G. Fabrizio, F. Colone, P. Lombardo, and A. Farina, "Adaptive beamforming for high-frequency over-the-horizon passive radar," IET Radar, Sonar \& Navigation, vol. 3, no. 4, pp. 384405, 2009.

[4] Y. Zhang, X. Li, and M. Amin, "Distributed beamforming in multi-user cooperative wireless networks," in Proceedings of the 4th International Conference on Communications and Networking in China (COM '09), pp. 1-5, August 2009.

[5] M. Elmer, B. D. Jeffs, K. F. Warnick, J. R. Fisher, and R. D. Norrod, "Beamformer design methods for radio astronomical phased array feeds," IEEE Transactions on Antennas and Propagation, vol. 60, no. 2, pp. 903-914, 2012.

[6] K. Gold, R. Silva, R. Worrel, and A. Brown, "Space navigation with digital beam steering GPS receiver technology," in Proceedings of the 59th Annual Meeting of The Institute of Navigation, Alberquerque, NM, USA, 2003.

[7] "Enhancing Access to Radio Spectrum (EARS)," National Science Foundation (NSF) solicitation 12-546, 2012, http:// www.nsf.gov/funding/pgm_summ.jsp?pims_id=503480.

[8] D. Cabric, "Cognitive Co-Existence in Heterogeneous Wireless NetworksCNS Division of Computer and Network Systems," February, 7, 2012.

[9] A. ] Madanayake, C. Wijenayake, N. Tran, S. V. Hum, L. T. Bruton, and T. Cooklev, "Directional spectrum sensing using tunable multi-D space-time discrete filters," in Procedings of the IEEE Workshop on Emerging Cognitive Radio Applications and Algorithms (CORAL '12), San Francisco, Calif, USA, 2012.

[10] H. L. P. A. Madanayake and L. T. Bruton, "A speed-optimized systolic array processor architecture for spatio-temporal 2-D IIR broadband beam filters," IEEE Transactions on Circuits and Systems I, vol. 55, no. 7, pp. 1953-1966, 2008.

[11] P. Agathoklis and L. T. Bruton, "Practical-BIBO stability of Ndimensional discrete systems," IEEE Proceedings G Electronic Circuits and Systems, vol. 130, no. 6, pp. 236-242, 1983.

[12] L. T. Bruton, "Three-dimensional cone filter banks," IEEE Transactions on Circuits and Systems I, vol. 50, no. 2, pp. 208216, 2003.

[13] L. T. Bruton and N. R. Bartley, "Highly selective threedimensional recursive beam filters using intersecting resonant planes," IEEE Transactions on Circuits and Systems, vol. 30, no. 3, pp. 190-193, 1983.

[14] K. K. Parhi and D. G. Messerschmitt, "Concurrent architectures for two-dimensional recursive digital filtering," IEEE
Transactions on Circuits and Systems, vol. 36, no. 6, pp. 813829, 1992.

[15] J. D. Kraus and R. J. Marhefka, Antennas for All Applications, McGraw-Hill, 2003.

[16] B. D. van Veen and K. M. Buckley, "Beamforming: a versatile approach to spatial filtering," IEEE ASSP magazine, vol. 5, no. 2, pp. 4-24, 1988.

[17] W. Liu and S. Weiss, Wideband Beamforming Concepts and Techniques, John Wiley \& Sons, 2010.

[18] J. Litva and T. K.-Y. Lo, Digital Beamforming in Wireless Communications, Artech House, Norwood, Mass, USA, 1996.

[19] L. C. Godara, "Application of antenna arrays to mobile communications, part II: beam-forming and direction-of-arrival considerations," Proceedings of the IEEE, vol. 85, no. 8, pp. 1195-1245, 1997.

[20] L. Liang and S. V. Hum, "Experimental characterization of UWB beamformers based on multidimensional beam filters," IEEE Transactions on Antennas and Propagation, vol. 59, no. 1, pp. 304-309, 2011.

[21] L. Liang and S. V. Hum, "Experimental verification of an adaptive UWB beamformer based on multidimensional filtering in a real radio channel," in Procedings of the IEEE International Symposium on Antennas and Propagation Society (APSURSI'10), pp. 1-4, July 2010.

[22] B. Veidt and P. Dewdney, "Bandwidth limits of beamforming networks for low-noise focal-plane arrays," IEEE Transactions on Antennas and Propagation, vol. 53, no. 1, pp. 450-454, 2005.

[23] C. T. Rodenbeck, S. G. Kim, W. H. Tu et al., "Ultrawideband low-cost phased-array radars," IEEE Transactions on Microwave Theory and Techniques, vol. 53, no. 12, pp. 36973702, 2005.

[24] E. Brookner, "Phased-array and radar breakthroughs," in Proceedings of the IEEE Conference on Radar, pp. 37-42, April 2007.

[25] E. Brookner, "Phased-Array and radar astounding breakthroughs - an update," in Proceedings of the IEEE Conference on Radar (RADAR '08), pp. 1-6, May 2008.

[26] M. A. Fischman, C. Le, and P. A. Rosen, "A digital beamforming processor for the joint DoD/NASA space based radar mission," in Proceedings of the IEEE Conference on Radar, pp. 9-14, April 2004.

[27] A. Brown and R. Silva, "GPS digital phased array antenna and receiver," in Proceedings of the IEEE International Conference on Phased Array Systems and Technology, pp. 153-156, May 2000.

[28] R. Fontana, A. Ameti, E. Richley, L. Beard, and D. Guy, "Recent advances in ultra wideband communications systems," in IEEE Conference on Ultra Wideband Systems and Technologies, Digest of Papers, pp. 129-133, 2002.

[29] C. G. Bilich, "Bio-medical sensing using Ultra Wideband communications and radar technology: a feasibility study," in Proceedings of the Pervasive Health Conference and Workshops, p. 19, November 2006.

[30] Y. Zhu and N. Xie, "An adaptive rake receiver for time hopping impulse radio ultra-wideband communications," in Proceedings of the Asia-Pacific Conference on Communications, pp. 371-375, October 2005.

[31] M. Ghavami, L. Michael, and R. Kohno, Ultra Wideband Signals and Systems in Communications Engineering, John Wiley \& Sons, 2004.

[32] X. Lian, H. Nikookar, and J. Zhou, "Adaptive robust beamformers for cognitive radio," in Proceedings of the 1st European Wireless Technology Conference (EUWIT '08), pp. 103-106, October 2008. 
[33] G. Zheng, S. Ma, K. K. Wong, and T. S. Ng, "Robust beamforming in cognitive radio," IEEE Transactions on Wireless Communications, vol. 9, no. 2, pp. 570-576, 2010.

[34] A. Petosa, "Frequency agile antennas for wireless communications-a survey," in Proceedings of the 14th International Symposium on Antenna Technology and Applied Electromagnetics \& the American Electromagnetics Conference (ANTEM/AMEREM '10), pp. 1-4, July 2010.

[35] T. Meehan, "The TriG digital beam steered sounder," in Proceedings of the IEEE Conference on Aerospace, pp. 1-5, 2009.

[36] Arnold van Ardenne, "Concepts of the square kilometre array; toward the new generation radio telescopes," IEEE International Symposium on Antennas and Propagation, vol. 1, pp. 158-161, 2000.

[37] G. Hampson, A. Smolders, and G. Kant, "Hierarchical beamforming aspects of OSMA," in Proceedings of the 5th International Symposium on Signal Processing and Its Applications (ISSPA '99), vol. 2, pp. 869-872, 1999.

[38] A. van Ardenne, "New generations of radio telescopes: antenna concepts and technologies," in Proceedings of the 12th International Conference on Antennas and Propagation (ICAP '03), vol. 2, pp. 526-529, 2003.

[39] J. Welch, D. Backer, L. Blitz et al., "The allen telescope array: the first widefield, panchromatic, snapshot radio camera for radio astronomy and SETI," Proceedings of the IEEE, vol. 97, no. 8, pp. 1438-1447, 2009.

[40] S. W. Ellingson, T. E. Clarke, A. Cohen et al., "The long wavelength array," Proceedings of the IEEE, vol. 97, no. 8, pp. 14211430, 2009.

[41] R. Armstrong, J. Hickish, K. Z. Adami, and M. Jones, "A digital broadband beamforming architecture for 2-PAD," in Wide Field Science and Technology For the Square Kilometre Array, pp. 284-288, 2009.

[42] K. Zarb-Adami, A. Faulkner, J. G. Bij de Vaate, G. W. Kant, and P. Picard, "Beamforming techniques for large- $\mathrm{N}$ aperture arrays," in Proceedings of the 4th IEEE International Symposium on Phased Array Systems and Technology (ARRAY'10), pp. 883890, October 2010.

[43] A.-J. van der Veen, A. Leshem, and A.-J. Boonstra, "Signal processing for radio astronomical arrays," in Proceedings of the Sensor Array and Multichannel Signal Processing Workshop, pp. 1-10, July 2004.

[44] G. W. Kant, P. D. Patel, S. J. Wijnholds, M. Ruiter, and E. van der Wal, "EMBRACE: a multi-beam 20,000-element radio astronomical phased array antenna demonstrator," IEEE Transactions on Antennas and Propagation, vol. 59, no. 6, pp. 1990-2003, 2011.

[45] K. F. Warnick, B. D. Jeffs, J. Landon et al., "Beamforming and imaging with the BYU/NRAO L-band 19-element phased array feed," in Proceedings of the 13th International Symposium on Antenna Technology and Applied Electromagnetics and the Canadian Radio Sciences Meeting (ANTEM/URSI '09), pp. 14, February 2009.

[46] J. B. Mead, "Digital beamforming receiver architectures for microwave remote sensing applications," in Proceedings of the IEEE International Geoscience and Remote Sensing Symposium (IGARSS '98), vol. 1, pp. 132-134, July 1998.

[47] M. Aamna, S. Ammar, T. Rameez, S. Shabeeb, N. I. Rao, and I. Safwat, "2D beamforming for through-the-wall microwave imaging applications," in Proceedings of the 2nd International Conference on Information and Emerging Technologies (ICIET '10), pp. 1-6, June 2010.
[48] R. Rüster, P. Czechowsky, P. Hoffmann, and W. Singer, "Gravity wave signatures at mesopause heights," Annales Geophysicae, vol. 14, no. 11, pp. 1186-1191, 1996.

[49] A. Smolders and M. van Haarlem, "Perspective on Radio Astronomy," in Proceedings of Technology for Large Antenna Arrays, Netherlands Foundation for research in Astronomy, 1999.

[50] A. van Ardenne, P. N. Wilkinson, P. D. Patel, and J. Bij de Vaate, "Electronic multi-beam radio astronomy concept: embrace a demonstrator for the European SKA program," Experimental Astronomy, vol. 17, no. 1-3, pp. 65-77, 2004.

[51] P. D. Patel, D. W. Kant, E. V. D. Wal, and A. van Ardene, "Phased array antennas demonstrator as a radio telescopeEMBRACE," in Proceedings of the IEEE International Symposium on Antennas and Propagation (AP-S '08), pp. 1-4, July 2008.

[52] M. Ruiter and E. van der Wal, "EMBRACE, a 10000 element next generation aperture array telescope," in Proceedings of the European Microwave Week Conference (EuMC '09), pp. 326329, September 2009.

[53] R. Mittra, "Square kilometer array-a unique instrument for exploring the mysteries of the universe using the square kilometer array," in Proceedings of the Applied Electromagnetics Conference (AEMC '09), pp. 1-6, December 2009.

[54] B. Gaensler, "The square kilometre array: an international radio telescope for the 21st century," in Proceedings of the IEEE International Symposium on Frequency Control, Joint with the 22nd European Frequency and Time Forum, April 2009.

[55] P. E. Dewdney, P. J. Hall, R. T. Schilizzi, and T. J. L. W. Lazio, "The square kilometre array," Proceedings of the IEEE, vol. 97, no. 8, pp. 1482-1496, 2009.

[56] A. Faulkner et al., "The Aperture Arrays for the SKA," The SKADS white paper, memo 122, 2010, http://www.skatelescope.org/.

[57] B. Veidt, G. J. Hovey, T. Burgess et al., "Demonstration of a dual-polarized phased-array feed," IEEE Transactions on Antennas and Propagation, vol. 59, no. 6, pp. 2047-2057, 2011.

[58] B. Veidt, T. Burgess, R. Messing, G. Hovey, and R. Smegal, "The DRAO phased array feed demonstrator: recent results," in Proceedings of the 13th International Symposium on Antenna Technology and Applied Electromagnetics and the Canadian Radio Sciences Meeting (ANTEM/URSI '09), pp. 1-4, February 2009.

[59] S. Weiss and I. K. Proudler, "Comparing efficient broadband beamforming architectures and their performance trade-offs," in Proceedings of the 14th International Conference on Digital Signal Processing (DSP '02), vol. 1, pp. 417-423, 2002.

[60] Y. Yang, C. Wan, and C. Sun, "Adaptive design of FIR filter with applications in broadband beamforming," in Proceedings of the IEEE Region 10 Conference (TENCON '04), pp. A507A510, November 2004.

[61] T. Sekiguchi, R. Miura, A. Klouche-Djedid, and Y. Karasawa, "Design of two-dimensional FIR digital filters used for broadband digital beamforming by combination of spectral transformation and window method," in Proceedings of the IEEE Region Digital Signal Processing Applications Conference (TENCON '96), vol. 1, pp. 261-266, November 1996.

[62] K. Houston, "A fast beamforming algorithm," in Proceedings of the Oceans Engineering for Today's Technology and Tomorrow's Preservation (OCEANS '94), vol. 1, pp. I/211-I/216, 1994.

[63] J.-N. Park, T. Usagawa, and M. Ebata, "Adaptive beamforming system using blind deconvolution with sharp mainlobe," in Proceedings of the IEEE Region 10 Conference (TENCON '99), vol. 1, pp. 538-554, 1999. 
[64] B. D. Jeffs, K. F. Warnick, J. Landon et al., "Signal processing for phased array feeds in radio astronomical telescopes," IEEE Journal on Selected Topics in Signal Processing, vol. 2, no. 5, pp. 635-646, 2008.

[65] M. Elmer and B. D. Jeffs, "Beamformer design for radio astronomical phased array feeds," in Proceedings of the IEEE International Conference on Acoustics, Speech, and Signal Processing (ICASSP '10), pp. 2790-2793, March 2010.

[66] M. de Vos, A. W. Gunst, and R. Nijboer, "The LOFAR telescope: system architecture and signal processing," Proceedings of the IEEE, vol. 97, no. 8, pp. 1431-1437, 2009.

[67] M. de Vos, "LOFAR: the first of a new generation of radio telescopes," in Proceedings of the IEEE International Conference on Acoustics, Speech, and Signal Processing (ICASSP '05), pp. V865-V868, March 2005.

[68] A. W. Gunst and M. J. Bentum, "Signal processing aspects of the low frequency Array," in Proceedings of the IEEE International Conference on Signal Processing and Communications (ICSPC '07), pp. 600-603, November 2007.

[69] L. Belostotski and J. W. Haslett, "A technique for differential noise figure measurement of differential LNAs," Proceedings of the IEEE Transactions on Instrumentation and Measurement, vol. 57, no. 7, pp. 1298-1303, 2008.

[70] A. Meaamar, C. C. Boon, K. S. Yeo, and M. A. Do, "A wideband low power low-noise amplifier in CMOS technology," IEEE Transactions on Circuits and Systems I, vol. 57, no. 4, pp. 773$782,2010$.

[71] L. Belostotski, J. W. Haslett, and B. Veidt, "Wide-band CMOS low noise amplifier for applications in radio astronomy," in Proceedings of the IEEE International Symposium on Circuits and Systems (ISCAS '06), 2006.

[72] A. Gunst, A. Kokkeler, and G. Kant, "A/D converter research for SKA," in Proceedings of the Perspectives on Radio Astronomy: Technologies for Large Antenna Arrays, pp. 261-264, Astron, Dingeloo, The Netherlands, 1999.

[73] G. L. Rosen, "ULA delay-and-sum beamforming for plume source localizationin," in Proceedings of the IEEE Workshop Signal Processing Applications for Public Security and Forensics (SAFE'07), pp. 1-4, 2007.

[74] J. Skudera and J. William, "SAW tapped delay lines for new potential circuit applications," in Proceedings of the 42nd Annual Frequency Control Symposium, pp. 252-258, 1988.

[75] J. T. Mayhan, A. J. Simmons, and W. C. Cummings, "Wideband adaptive antenna nulling using tapped delay lines," IEEE Transactions on Antennas and Propagation, vol. 29, no. 6, pp. 923-936, 1981.

[76] W. Wen, L. Ning, T. Jun, and P. Yingning, "Broadband digital beamforming based on fractional delay in SAR systems," in Proceedings of the 2nd Asia-Pacific Conference on Synthetic Aperture Radar (APSAR '09), pp. 575-578, October 2009.

[77] H. Iwakura, "Realization of tapped delay lines using switchedcapacitor LDI ladders and application to FIR filter design," IEEE Transactions on Circuits and Systems II, vol. 40, no. 12, pp. 794-797, 1993.

[78] J. G. Proakis and D. G. Manolakis, Digital Signal Processing Principles, Algorithms, and Aplications, Pearson Prentice Hall, New Jersey, NJ, USA, 1996.

[79] D. E. Dudgeon and R. M. Mersereau, Multidimensional Digital Signal Processing, Prentice Hall, Englewood Cliffs, NJ, USA, 1990.

[80] S. V. Hum, H. L. P. A. Madanayake, and L. T. Bruton, "UWB beamforming using 2-D beam digital filters," IEEE Transactions on Antennas and Propagation, vol. 57, no. 3, pp. 804-807, 2009.
[81] D. E. Dudgeon and R. M. Mersereau, Multidimensional Digital Signal Processing, Prentice Hall, Englewood Cliffs, NJ, USA, 1984.

[82] A. Madanayake and L. T. Bruton, Radio-Frequency (RF) Beamforming Using Systolic FPGA Based Two-Dimensional 2D IIR Space-Time Filters, InTech, 2010.

[83] A. I. Zerev, Handbook of Filter Synthesis, Wiley-Interscience, 1st edition, 1967.

[84] N. Ganganath, G. Attanayake, T. Y. Bandara et al., "Scannedarray audio beamforming using 2nd- and 3rd-order 2D IIR beam filters on FPGA," in Proceedings of the International Conference on Microelectronics (ICM '10), pp. 451-454, December 2010.

[85] L. T. Bruton, RC-Active Circuits Theory and Design, Prentice Hall, Englewood Cliffs, NJ, USA, 1980.

[86] K. K. Parhi, VLSI Digital Signal Processing Systems, Wiley, India, 2007.

[87] K. K. Parhi and D. Messerschmitt, "Look-ahead computation: improving iteration bound in linear recursions," in Proceedings of the IEEE International Conference on Acoustics, Speech, and Signal Processing (ICASSP '87), pp. 1855-1858, 1987.

[88] H. L. P. A. Madanayake and L. T. Bruton, "Time-multiplexed systolic-array processors for real-time 2D IIR beam planewave filters," in Proceedings of the 50th Midwest Symposium on Circuits and Systems (MWSCAS '07), pp. 686-689, August 2007.

[89] A. Madanayake, L. T. Bruton, and C. Comis, "FPGA architectures for real-time 2D/3D FIR/IIR plane wave filters," in Proceedings of the IEEE International Symposium on Cirquits and Systems (ISCAS '04), vol. 3, May 2004.

[90] M. G. M. Hussain, "Principles of space-time array processing for ultrawide-band impulse radar and radio communications," IEEE Transactions on Vehicular Technology, vol. 51, no. 3, pp. 393-403, 2002.

[91] M. J. Flynn, "Area-Time-Power and design effort: the basic tradeoffs in application specific systems," in Proceedings of the IEEE 16th International Conference on Application-Specific Systems, Architectures, and Processors (ASAP'05), pp. 3-5, July 2005. 

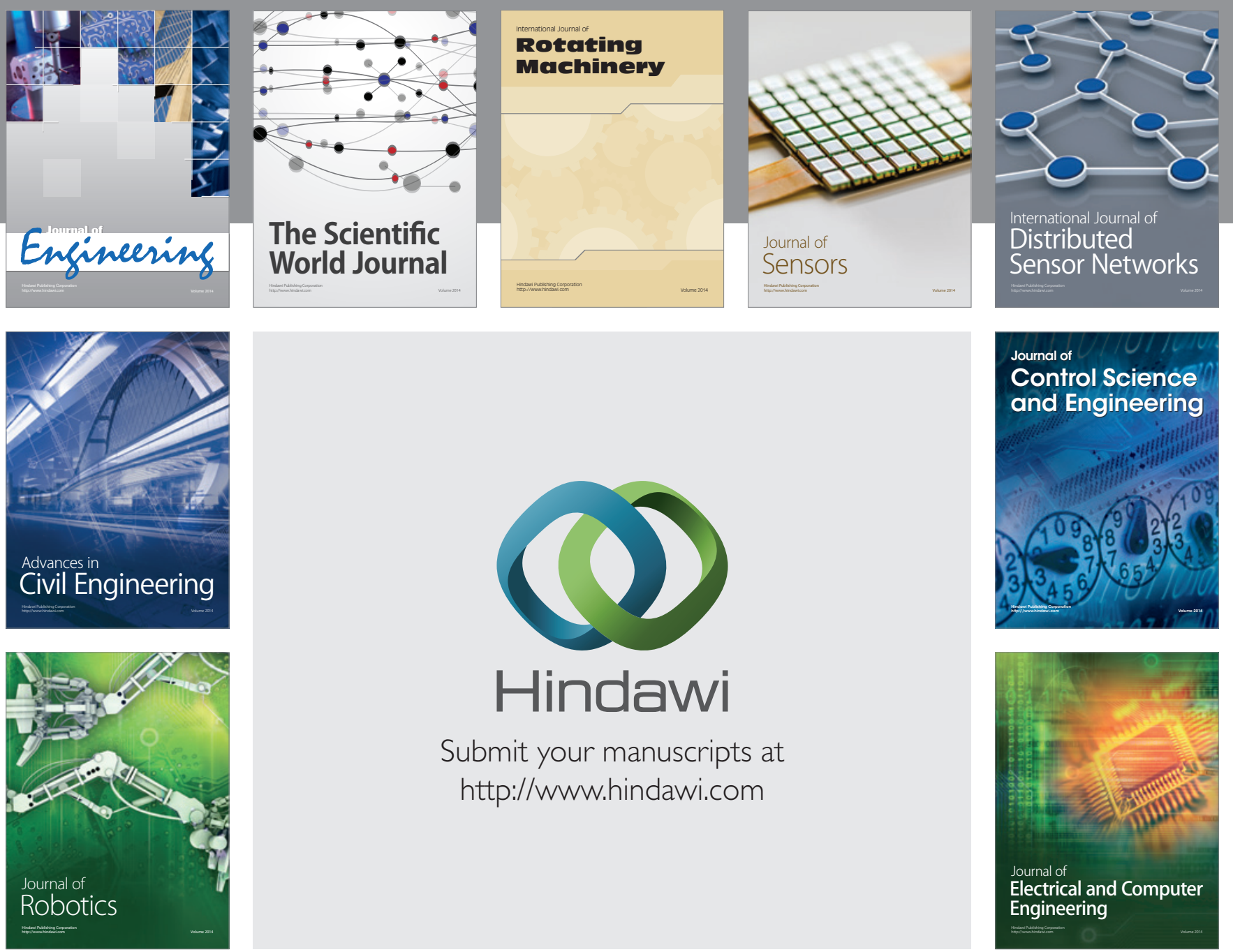

Submit your manuscripts at

http://www.hindawi.com
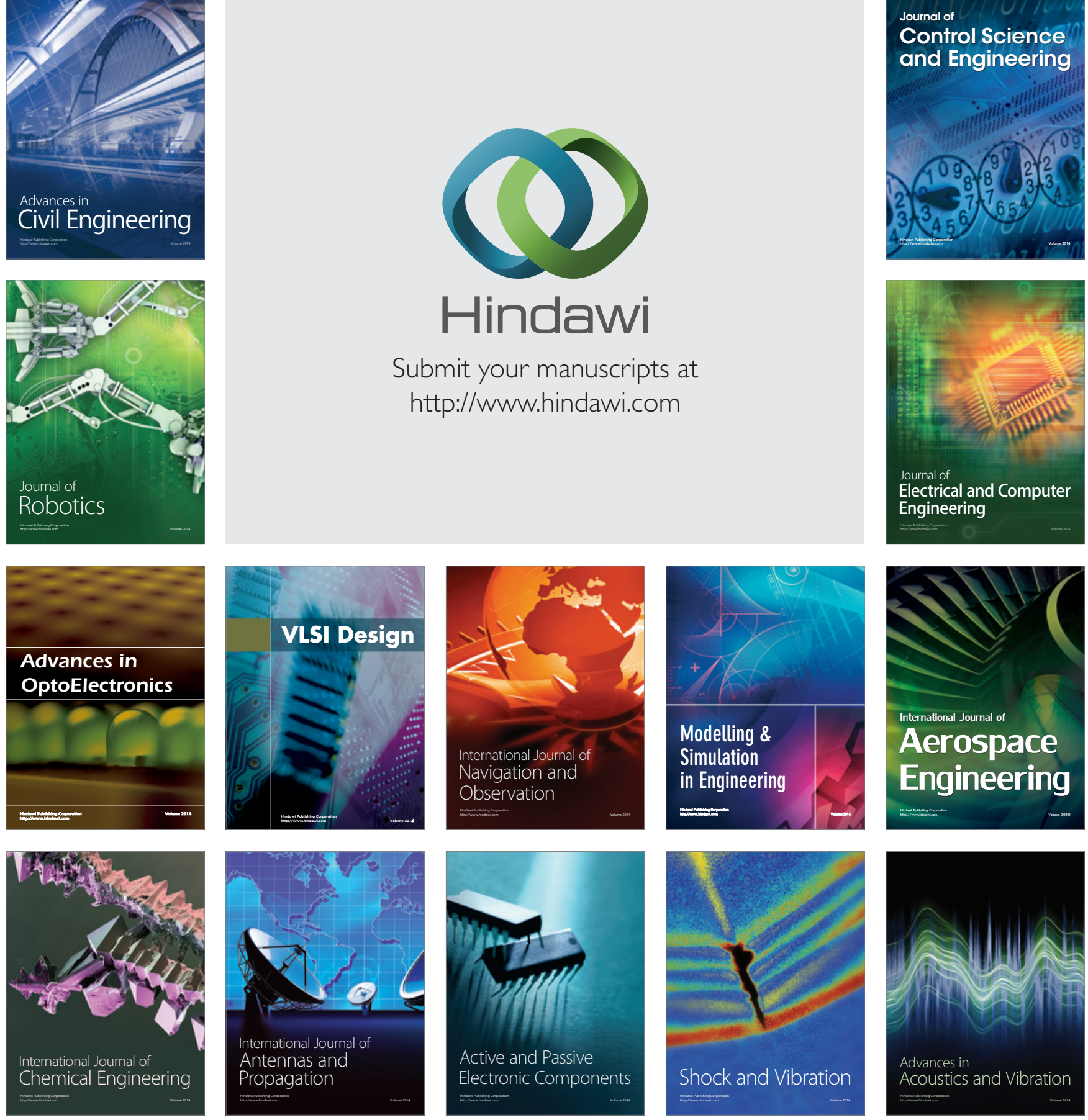